\title{
Charakteristika von durch den Innovationsfonds geförderten Interventionsstudien: Review und Dokumentenanalyse von Studienprotokollen, Publikationen und Abschlussberichten
}

\section{Characteristics of Innovation Fund-supported Intervention Studies: Review and Document Analysis of Study Protocols, Publications and Final Reports}

\author{
Autoren \\ Heike Heytens ${ }^{1}$, Felix Walther ${ }^{2}, 3$, Laura Keßler ${ }^{5}$, Daniel Bremer ${ }^{4}$, Elisa Frenz² ${ }^{2}$ Martin Härter ${ }^{4}$, Max Geraedts ${ }^{6}$, Thomas \\ Bierbaum $^{5}$, Christian Apfelbacher ${ }^{1}$, Jochen Schmitt ${ }^{2}$
}

Institute

1 Institut für Sozialmedizin und Gesundheitssystemforschung, Otto-von-Guericke-Universität Magdeburg, Magdeburg

2 Zentrum für Evidenzbasierte Gesundheitsversorgung, Universitätsklinikum und Medizinische Fakultät Carl Gustav Carus an der Technischen Universität, Dresden

3 Qualitäts- und Medizinisches Risikomanagement, Universitätsklinikum Carl Gustav Carus Dresden, Dresden

4 Institut und Poliklinik für Medizinische Psychologie, Center for Health Care Research, Universitätsklinikum Hamburg-Eppendorf, Hamburg

5 Deutsches Netzwerk Versorgungsforschung e. V., Berlin

6 Institut für Versorgungsforschung und Klinische Epidemiologie, Philipps-Universität Marburg, Marburg

Schlüsselwörter

Innovationsfonds, Interventionsstudien, RCT, Review

Keywords

innovation fund, intervention study, RCT, review

Bibliografie

Gesundheitswesen 2021; 83: e20-e39

DOI $10.1055 / a-1448-2412$

ISSN 0941-3790

(c) 2021. Thieme. All rights reserved.

Georg Thieme Verlag KG, Rüdigerstraße 14,

70469 Stuttgart, Germany

Korrespondenzadresse

Dr. Thomas Bierbaum

Deutsches Netzwerk Versorgungsforschung e. V.

Kuno-Fischer-Straße 8

14057 Berlin

Deutschland

tbierbaum@dnvf.de

\section{ZUSAMMENFASSUNG}

Dieses prospektiv registrierte Review charakterisiert 50 Interventionsstudien der ersten Welle des Innovationsfonds auf Grundlage von Studienprotokollen oder Originalartikeln. Die mehrheitlich (randomisierten) kontrollierten Interventionsstudien bezogen überwiegend Erwachsene bzw. Senioren in ambulanter Versorgung ohne regionalen Fokus ein und analysierten Behandlungsprozesse mit heterogenen klinischen und Patientenoutcomes als Zielgrößen. Die zu einem substantiellen Teil fehlenden Studienprotokolle und methodischen Details (u. a. Fallzahlplanung) zeigen (vermeidbare) methodische Probleme hinsichtlich der wissenschaftlichen Qualität der geförderten Studien auf.

\begin{abstract}
This prospectively registered review characterizes 50 intervention studies from the $1^{\text {st }}$ wave of the Innovation Fund based on study protocols or original articles, among other sources. The mainly (randomized) controlled intervention studies included predominantly adults/seniors in ambulatory care without a regional focus and analyzed treatment processes, clinical and patient outcomes as outcomes. The substantial lack of study protocols and methodological details (e. g., sample size planning) reveals (avoidable) methodological problems regarding the scientific quality of the funded studies.
\end{abstract}




\section{Einleitung}

Im Jahr 2015 wurde mit der Verabschiedung des Gesetzes zur Stärkung der Versorgung in der gesetzlichen Krankenversicherung (GKV-Versorgungsstärkungsgesetz) die Grundlage für den Innovationsfonds gelegt. Angesiedelt beim Gemeinsamen Bundesausschuss (G-BA) und ausgestattet mit einem jährlichen Fördervolumen von derzeit 200 Mio. Euro (2016-2019 je 300 Mio.) soll der Innovationsfonds durch die Entwicklung neuer Versorgungskonzepte eine versorgungswissenschaftlich evaluierte Weiterentwicklung der umlagefinanzierten Gesundheitsversorgung erzielen. Damit gehört der Innovationsfonds weltweit zu einem kleinen Kreis ähnlicher Initiativen mit vergleichbarem Zweck [1-4]. Aus Sicht des Deutschen Netzwerks Versorgungsforschung (DNVF) besteht damit die große Chance, die deutsche Gesundheitsversorgung evidenzbasiert weiterzuentwickeln und zukunftsfähig zu gestalten [5]. Zum Start des Innovationsfonds wurden in einer Delphi-Studie des DNVF interprofessionell und interdisziplinär konsentierte Empfehlungen für prioritäre Themenfelder, Anforderungen an die Methodik und an die Ergebniskommunikation an den Gemeinsamen Bundesausschuss und alle anderen Interessengruppen kommuniziert und publiziert [6]. Unbekannt ist bisher, inwieweit die geförderten Projekte diese Empfehlungen berücksichtigt bzw. umgesetzt haben. Die 2020 begründete Ad-hoc-Kommission „Innovationfonds“ des DNVF hat deshalb zum Ziel, die Qualität und das Potenzial der durch den Innovationsfonds geförderten Projekte in mehreren Teilstudien zu erfassen, zu überprüfen und zu veröffentlichen. In der vorliegenden Arbeit präsentieren wir erste Ergebnisse eines inhaltlichen und methodischen Reviews von Innovationsfonds-Projekten der ersten Förderwelle.

\section{Methodik}

Für dieses Review wurden ausschließlich Interventionsstudien der ersten Förderwelle eingeschlossen (Förderbekanntmachung vom 08.04.2016). Dem lag die Annahme zugrunde, dass inzwischen Abschlussberichte in großer Zahl verfügbar und somit eine möglichst

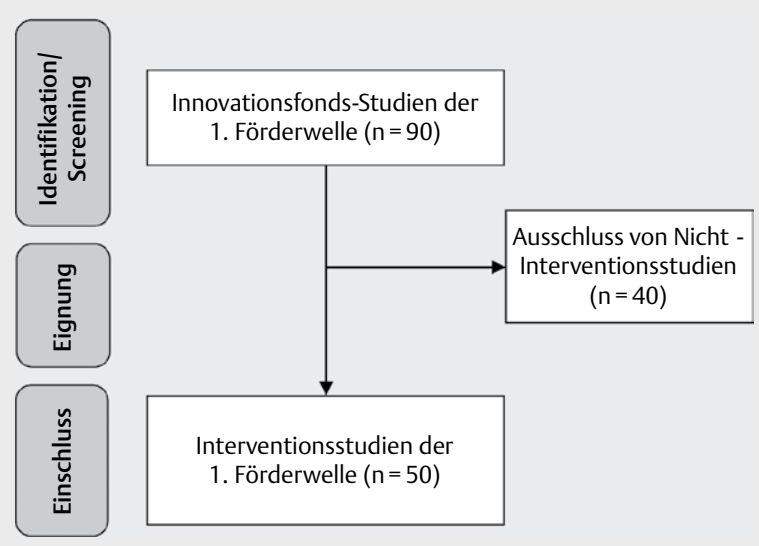

Abb. 1 Prisma Flow Chart. Quelle: Prisma: From: Moher D, Liberati A, Tetzlaff J, Altman DG, The PRISMA Group (2009). Preferred Reporting Items for Systematic Reviews and Meta-Analyses: The PRISMA Statement. PLoS Med 6(7): e1000097. doi:10.1371/journal. pmed1000097 vollständige qualitative Bewertung der wissenschaftlichen Güte und Ergebnisse der Projekte möglich sein sollten. In Anlehnung an die Definition der Weltgesundheitsorganisation (WHO) betrachten wir „Intervention“ als eine geplante und unter Studienbedingungen durchgeführte Veränderung von (Behandlungs)Prozessen zur Ergebnisverbesserung [7]. Gemäß dem Deutschen Register Klinischer Studien erachten wir als Interventionsstudie eine Maßnahme oder Reihe von Maßnahmen, mit dem Ziel einen Prozess, Handlungsablauf oder eine Folge von Ereignissen zu modifizieren, um eine oder mehrere ihrer Charakteristika wie z. B. Performanz, erwartete Outcomes, Output oder Struktur zu verändern [8]. Die Daten zur Einordnung der Förderwellen wurden von der Firma Ordinary People - Market Intelligence $\mathrm{GmbH}$ zur Verfügung gestellt und durch die Autoren gemäß der Förderbekanntmachung vom 08. April 2016 gegengeprüft. Einschränkungen bei Population, Intervention oder Outcome lagen nicht vor. Das Reviewprotokoll wurde prospektiv bei PROSPERO (https://www.crd.york.ac.uk/prospero/display_record.php?RecordID $=180223$ ) registriert und ohne Abweichungen durchgeführt. Screening und Einschluss erfolgten doppelt unabhängig mit Konsentierung in der Autorengruppe bei Dissens. Für die von November 2020 bis Januar 2021 via MS Excel durchgeführte Datenextraktion definierte und pilotierte das Autorenteam a-priori Evidenztabellen unter Berücksichtigung der Komponenten des PICOS-Schemas:

- Population (u. a. Altersgruppe, Indikation, regionales Setting),

- Intervention (u. a. Benennung, Zuordnung zu den Versorgungssektoren, beteiligte Berufsgruppen),

- Comparator/Kontrolle,

- Outcome (Primär- und Sekundäroutcomes),

- Studientyp.

Informationen aus Studienprotokollen (u.a. Sprache), Publikationen (u. a. Publikationstyp) und methodische Inhalte (u. a. Datenquellen, Fallzahlplanungen) wurden in der Pilotierungsphase doppelt unabhängig, später als Einfachextraktion mit Überprüfung extrahiert. Bei Dissens entschied ein dritter Reviewer. Mit letztmaliger Aktualisierung am 15.12.20 durchsuchte das Team im Rahmen eines a-priori festgelegten Verfahrens folgende Datenquellen:

- Projektbeschreibungen auf der Internetseite des Gemeinsamen Bundesausschuss (G-BA)

- vorhandene Studienregistrierungen (

- mehrstufige Publikationssuche in verschiedenen Datenbanken ( Abb. 3)

- Projektwebseiten

- vorhandene Abschlussberichte auf der Internetseite des G-BA.

\section{Ergebnisse}

Von 90 geförderten Projekten der ersten Welle des Innovationsfonds wurden gemäß o.g. Kriterien 50 Projekte als Interventionsstudien klassifiziert und in die Untersuchung eingeschlossen ( Abb. 1). 29 Projekte gehörten der Kategorie „Neue Versorgungsformen“, 21 der „Versorgungsforschung“ an. Das gesamte Fördervolumen dieser 50 Projekte betrug insgesamt ca. 234 Mio. $€$.

Eine zuvor festgelegte Suchstrategie in den einschlägigen Studienregistern, wie u. a. dem Deutschen Register Klinischer Studien 


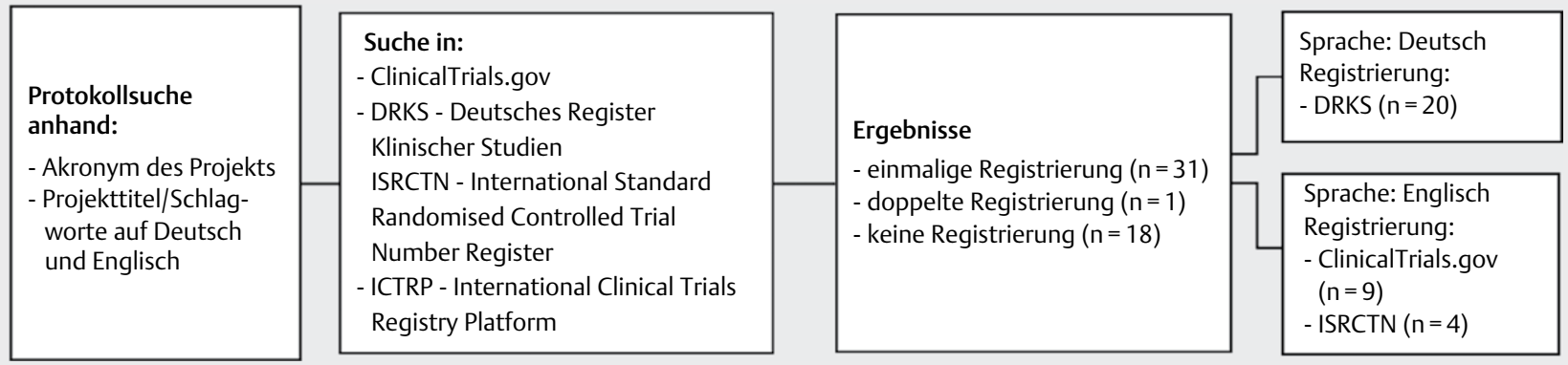

Abb. 2 Suchvorgang und Ergebnis zu registrierten Studienprotokollen

\begin{tabular}{|l|l|}
\hline Publikationssuche & \multicolumn{1}{|l|}{ Suche in: } \\
anhand: & - Thieme Connect \\
- Akronym des Projekts & - SpringerLink \\
- Projekttitel/Schlagworte & - ResearchGate \\
des Projekttitels auf & - German Medical \\
Deutsch und Englisch & Science \\
- Nummer der & - PubMed \\
Studienregistrierung & - Google \\
- Konsortialführer & - Google Scholar \\
\hline
\end{tabular}

Abb. 3 Ergebnis der Publikationssuche

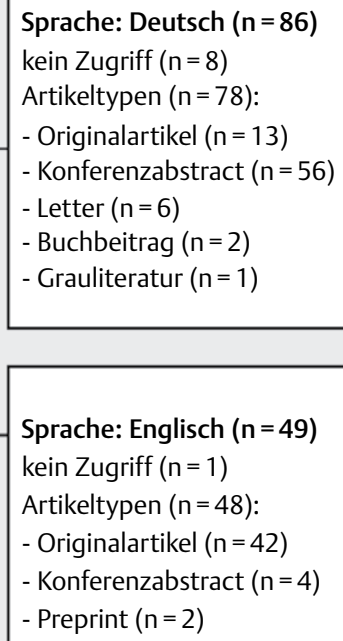

Publikationsinhalte $(n=78)$ :

- Protokoll $(n=40)$

- Analyseplan $(n=1)$

- Baseline $(n=3)$

- Sekundärergebnisse $(n=28)$

- Primärergebisse $(n=2)$

- Vorstudie $(n=1)$

- Allgemeine Informationen $(n=3)$

Publikationsinhalte $(n=48)$ :

- Protokoll $(n=24)$

- Protokoll + Baseline $(n=1)$

- Analyseplan $(n=1)$

- Sekundärergebnisse $(n=21)$

- Primärergebnisse $(n=1)$
(DRKS) und clinicaltrials.gov, führte bei etwa zwei Drittel $(n=32 / 50)$ der eingeschlossenen Projekte zu registrierten Studienprotokollen ( $\triangleright$ Abb. 2), d. h. bei 36 \% aller Studien konnten keine registrierten Studienprotokolle recherchiert werden.

Die Publikationssuche ( $\triangleright$ Abb. 3 ) identifizierte 135 veröffentlichte Artikel (davon 126 abrufbar) in (mehrheitlich) deutscher ( $n=86 / 135)$ und englischer $(n=49 / 135)$ Sprache. Auf Deutsch wurden hauptsächlich Konferenzabstracts $(n=56 / 86)$ und auf Englisch Originalartikel $(n=42 / 49)$ publiziert. Die 126 Artikel beinhalteten nur vereinzelt primäre Studienergebnisse $(n=3 / 126)$, größtenteils dagegen Studienprotokolle $(n=67 / 126)$ oder Sekundärergebnisse ( $n=49 / 126)$. Dabei konnten 6 Projekte bisher keine Publikationen vorweisen, 28 Projekte veröffentlichten bisher zwischen einer und drei Publikation, 13 zwischen vier und sechs Publikationen. Jeweils ein Projekt hatte bisher 7 bzw. 8 Veröffentlichungen. Zum 15.12.2020 waren gemäß der Internetseite des G-BA fünf Abschlussberichte abrufbar, 26 befanden sich in Erstellung, bei 19 Projekten wurde mit der Erstellung des Abschlussberichts noch nicht offiziell begonnen.
Hinsichtlich der vom G-BA vorgegebenen Themenfelder erstrecken sich die Projekte auf die Bereiche „Themenoffen“ ( $n=11)$, „spezielle Patientengruppen“ ( $n=10)$, „Qualität“ $(n=6)$, „Telemedizin“ ( $n=6)$ und weitere für z. B. den „ländlichen Raum“ oder „Medikamente“ ( $n=17)$. Eine detaillierte Charakterisierung in Anlehnung an das PICOS-Schema ist in \ Tab. 1 projektindividuell aufgeführt und folgend zusammengefasst.

\section{Auswertung gemäß PICOS-Schema}

\section{$\mathrm{P}=$ Population}

Rund die Hälfte der Studien (24/50) schlossen ausschließlich Erwachsene und/oder Senioren, 4 ausschließlich Kinder und Jugendliche und 22 Projekte (44\%) gemischte Altersgruppen ein. Konkrete geschlechtsspezifische Interventionen wurden nicht untersucht. Es gab bei der Mehrzahl der Studien ( $n=28 / 50)$ keinen explizit städtischen oder ländlichen Schwerpunkt. Die verbleibenden 22 Projekte untersuchten den ländlichen $(n=7)$, städtischen $(n=8)$ oder 


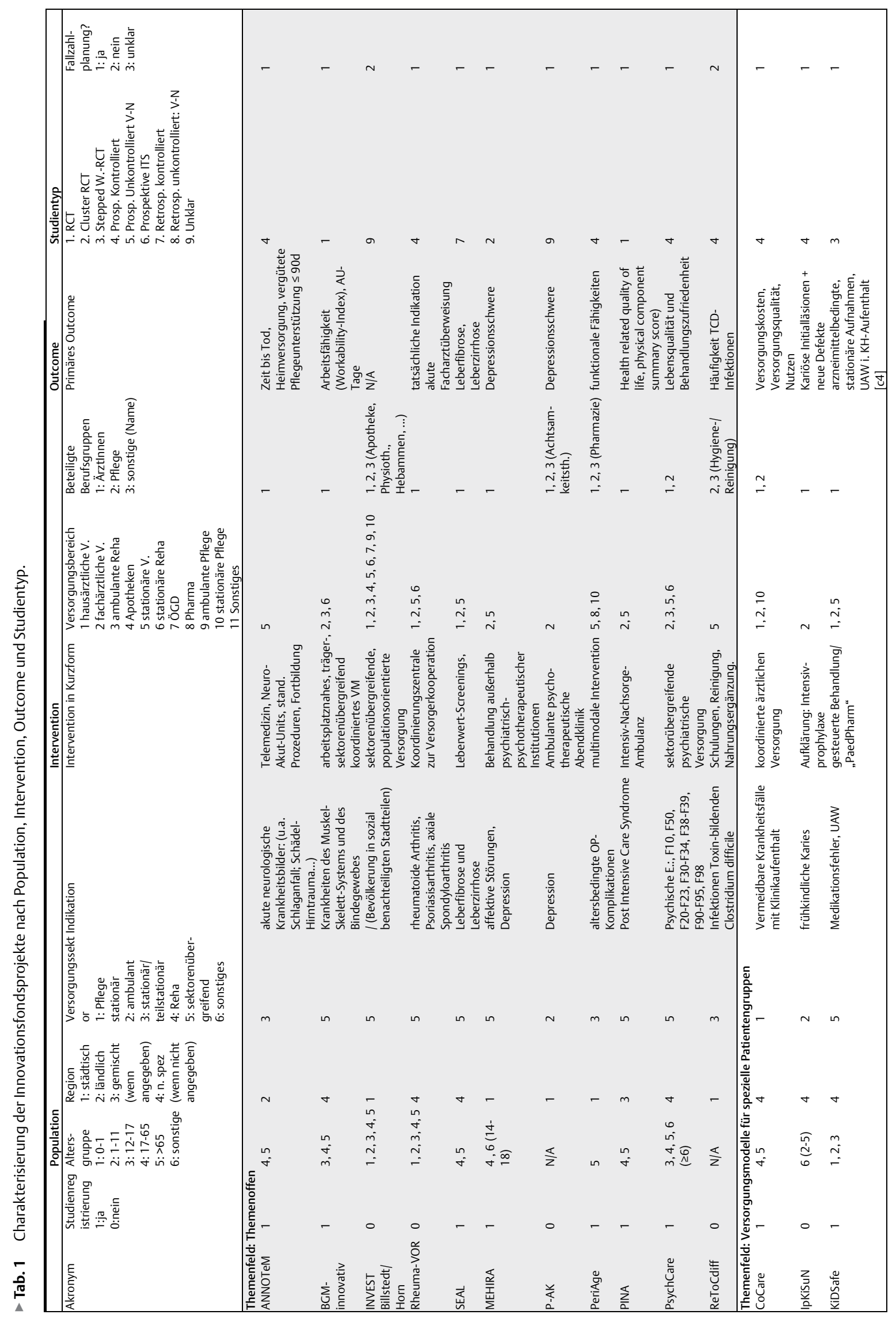




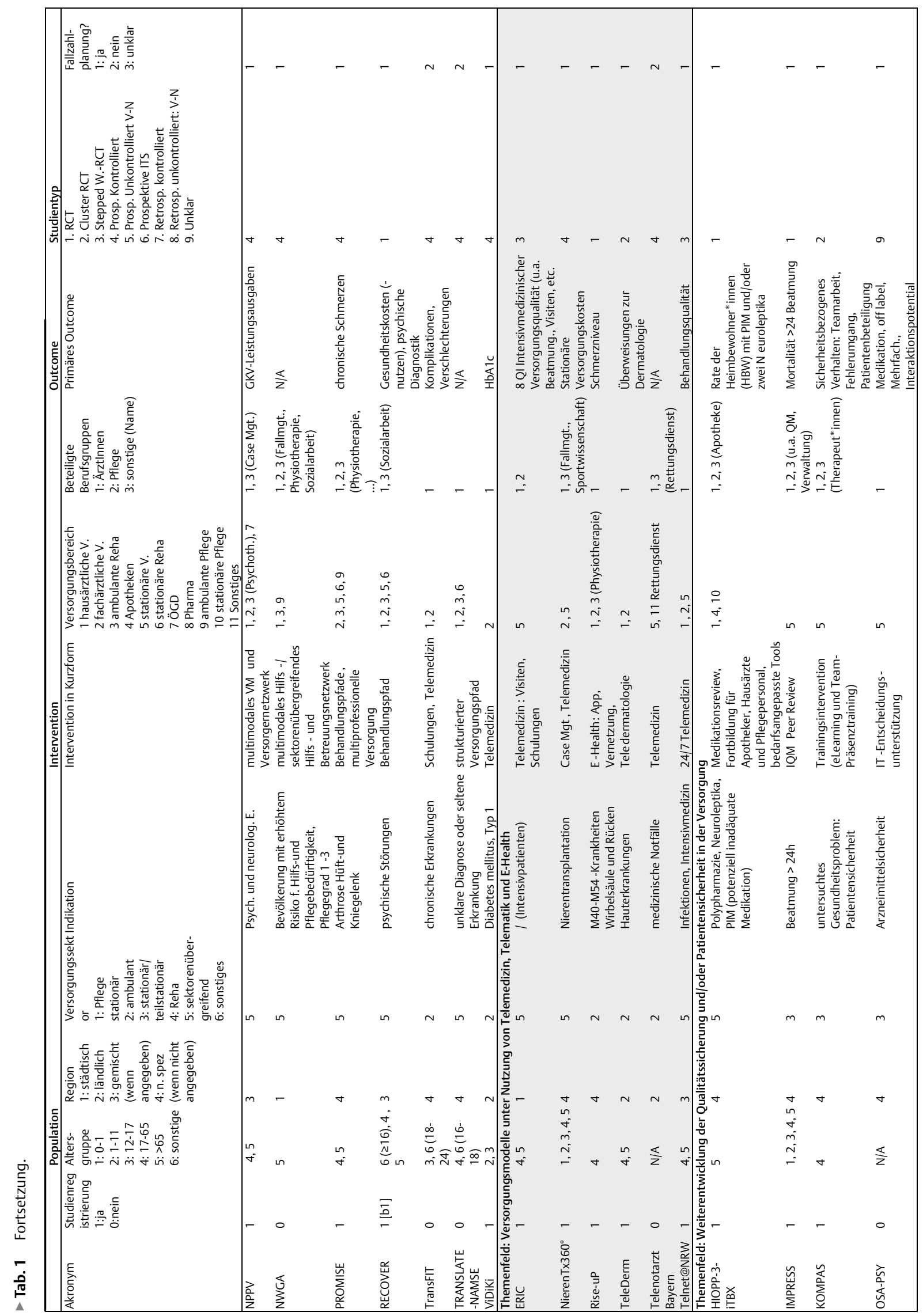




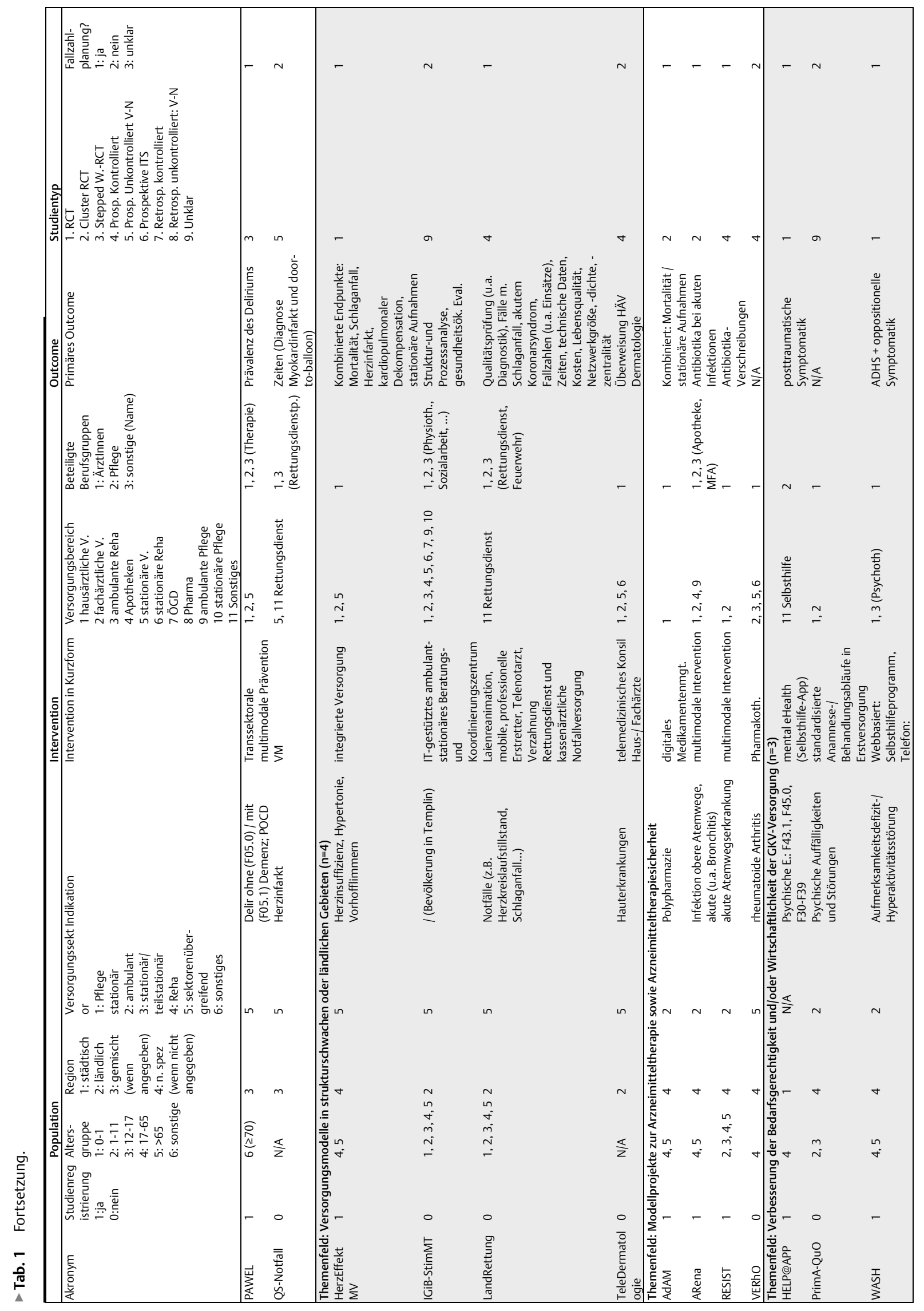







beide ( $n=7)$ Agglomerationsräume. Ebenso war die Mehrheit der Projekte $(n=27 / 50)$ sektorenübergreifend ausgerichtet. Ein ausschließlicher Fokus auf den ambulanten ( $n=13 / 50)$ oder (teil)stationären Gesundheitsversorgungs- $(n=6 / 50)$ oder Pflegesektor ( $n=2 / 50$ ) war nur bei wenigen Projekten der Fall. Mangels Detailinformationen ließen sich 2 Projekte bezüglich der untersuchten Sektoren nicht beschreiben. Im Hinblick auf die Indikation wurden in 19 von 50 Projekten keine Erkrankungen, sondern Versorgungssettings definiert (z. B. Pflegeheim in den Projekten IGiB-StimMT oder INVEST Billstedt/Horn, Notfälle, Intensivmedizin, Transplantationschirurgie). Die verbliebenen 32 Projekte analysierten dagegen unterschiedliche Erkrankungen, u. a. psychische Erkrankungen $(n=11)$, Erkrankungen des Muskel-Skelett-Systems ( $n=5)$, Infektionen $(n=4)$ oder neurologische Erkrankungen $(n=3)$.

\section{I = Intervention}

Bei der Durchführung der Interventionen waren bei rund drei Viertel $(n=37 / 50)$ mehrere Versorgungsbereiche beteiligt. Ihr primärer Fokus lag dabei häufig auf ambulanter haus- $(n=27)$ und fachärztlicher ( $n=33$ ) Versorgung, stationärer Akutversorgung $(n=27)$, seltener auf ambulanter $(n=13)$ oder stationärer $(n=11)$ Rehabilitation sowie ambulanter $(n=6)$ und stationärer $(n=7)$ Pflege. Andere Leistungsbereiche wie Rettungsdienst $(n=3)$, Apotheken $(n=5)$ oder der öffentliche Gesundheitsdienst $(n=4)$ waren vereinzelt an Interventionen beteiligt.

Hauptbeteiligte Berufsgruppen an den Interventionen waren Ärztinnen und Ärzte $(n=47 / 50)$ und/oder Pflegepersonal $(n=19 / 50)$. Weitere Berufsgruppen umfassten u. a. Therapeuten, Rettungsdienst- oder Apothekenpersonal $(n=23)$. Im Wesentlichen ( $n=44$ ) bestand die Projektplanung und -durchführung aus komplexen Ansätzen in Form mehrerer Sektoren, Berufsgruppen und verschiedenen Interventionsbestandteilen.

\section{$\mathrm{C}=$ Comparator/Kontrolle}

Bei 42 Projekten (84\%) bestand die Vergleichsgruppe zur Interventionsgruppe aus der Regelversorgung. Bei 7 Projekten fehlten Angaben zur Vergleichsgruppe, eine Studie hatte keine Vergleichsgruppe.

\section{$\mathrm{O}=$ Outcome}

In den untersuchten 50 Projekten wurde 72 sehr heterogene Outcomes definiert und den folgenden Dimensionen bzw. Bereichen zugeordnet ( $\mathbf{A b b}$. 4):

- Behandlungsstruktur, -prozess und -zufriedenheit (z. B. Verzahnung, Kommunikation, Behandlungsverlauf) $(n=15)$

- Patientenrelevante Outcomes $(n=15)$ (z. B. Lebensqualität $(n=5)$, kombinierte Endpunkte $(n=3)$ oder Mortalität $(n=1))$

- Erkrankungsspezifische/Klinische Diagnostik ( $n=11)$ (z. B. Depressionsscores, psychische Ausnahmesituationen, Blutparameter)

- Gesundheitsökonomische Outcomes $(n=8)$ (z. B. Kosten, Kosten-Nutzen oder Nutzenbewertungen)

- Inanspruchnahme/Leistungsgeschehen $(n=7)$ (z. B. stationäre Aufnahmen)

- Medikation $(n=6)$

- Qualität (z. B. Dokumentations- oder Diagnosequalität) $(n=4)$
Bei 8 Projekten ließen sich die Outcomes aufgrund fehlender Informationen nicht klassifizieren. Als primäre Datenquellen kamen mehrheitlich Befragungsdaten ( $n=17$ ) solitär oder mit klinischen Daten kombiniert $(n=24)$ zum Einsatz. Sekundärdaten umfassten weitgehend Abrechnungs- und Verordnungsdaten $(n=30)$ mit Fokus auf klinischen Outcomes oder Verordnungs- und Inanspruchnahmedaten.

\section{$S=$ Studientyp}

Den geförderten Studien lagen hauptsächlich randomisierte ( $n=20 / 43$ ) oder prospektiv nicht-randomisierte kontrollierte ( $n=20 / 43$ ) Designs zugrunde. 2 Studien waren retrospektiv kontrolliert, eine war nicht kontrolliert (Vorher-Nachher-Design). Bei 7 Projekten fehlten notwendige Informationen zur Klassifikation. Eine definierte Fallzahlplanung gaben 38 von 50 Studien an. Aussagen, ob eine Anpassung oder Erreichung der gewünschten Fallzahl vorlag, sind mangels vollständiger Studien-/ Ergebnisberichte und Publikationen bei einem Großteil $(n=31)$ der durchgeführten Projekte nicht möglich.

\section{Zusammenfassung und Diskussion}

Das Ziel dieser Arbeit war die qualitative Analyse und Charakterisierung geförderter Projekte und das daraus resultierende mögliche Transferpotenzial. Demgegenüber beurteilt das vom Bundesgesundheitsministerium in Auftrag gegebene quantitativ orientierte PROGNOS-Gutachten [9] ökonomische Aspekte in Form der Organisation des Innovationsfonds, Projektcontrolling sowie die Effektivität des Zusammenspiels der Gremien [10].

Die Charakterisierung von 50 geförderten Interventionsstudien der ersten Welle ergab in 36\% der Fälle ein fehlendes registriertes Studienprotokoll. Darüber hinaus waren deutlich weniger Abschlussberichte und Ergebnispublikationen verfügbar als anfangs angenommen. Dies führte aufgrund fehlender oder unvollständiger Informationen zu erheblichen Problemen bei der Beurteilung und Extraktion. Zahlreiche themenoffene Projekte lassen auf eine starke Abweichung von den vom G-BA festgelegten Schwerpunkten schließen. Insbesondere die geringe Beforschung der Gesundheitsversorgung von Kindern und Jugendlichen sowie eine adäquate Gesundheitsversorgung strukturschwacher und ländlich geprägter Regionen begründen diese Annahme. Abseits dessen zeugen häufig vorkommende interdisziplinäre und intersektorale Projekte davon, dass die Projektverantwortlichen das Dauerproblem einer traditionellen sektoralen Trennung des Gesundheitswesens [11] überwinden und eine bessere Verzahnung erreichen möchten. Gleiches trifft für zahlreiche Projekte zu, die Behandlungsstrukturen und -prozesse untersuchen oder das Patientenoutcome in den Mittelpunkt des Forschungsinteresses rücken.

Positiv heben sich insgesamt 40/43 randomisierte bzw. kontrollierte Studien zu überwiegend komplexen Interventionen hervor. Kritisch ist allerdings der Befund, dass bei $23 \%$ der 50 Projekte Angaben zur Fallzahlplanung und in 7 Fällen Angaben zu Vergleichsgruppen fehlten.

Allein das Fehlen registrierter Studienprotokolle führt zu mangelnder Transparenz, indem wichtige Studiendetails nicht ermittelt werden können - beispielsweise Rekrutierungsstrategien, Studiendesigns oder Outcomes. Zusätzlich wird dadurch eine qualita- 


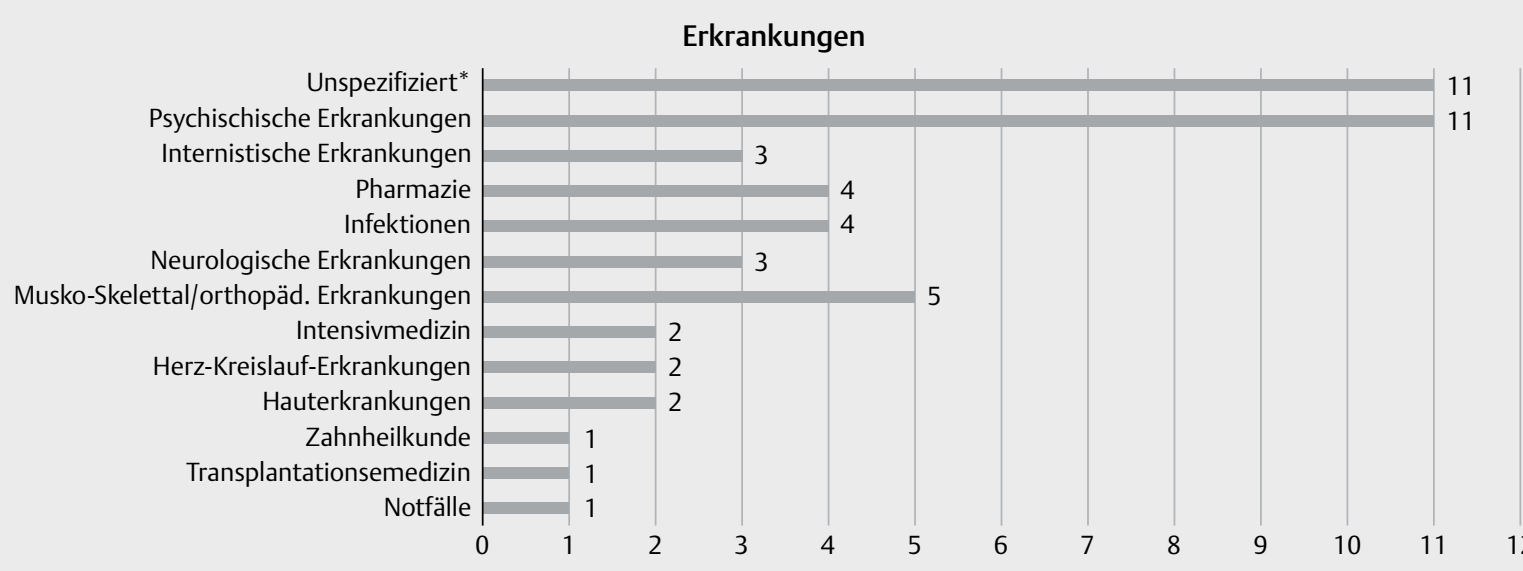

Zielregion

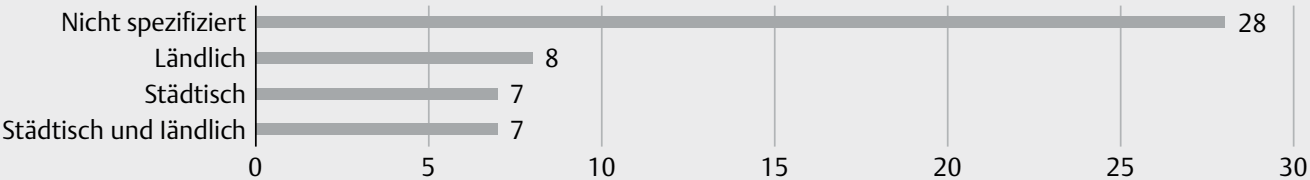

sektorale Zuordnung der Interventionen

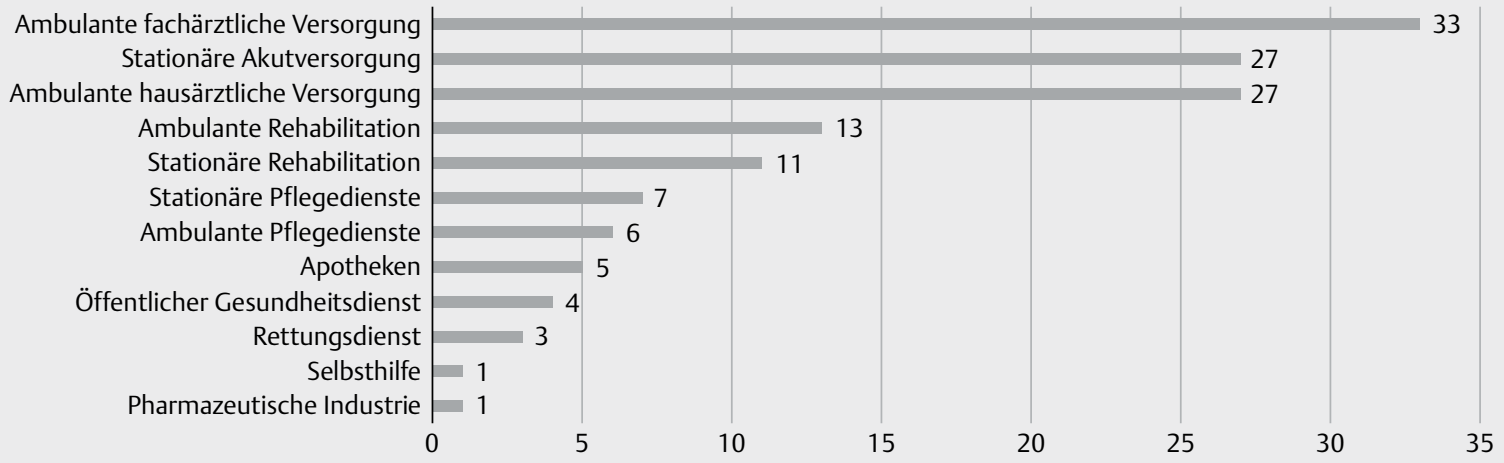

Outcome-Kategorisierungen

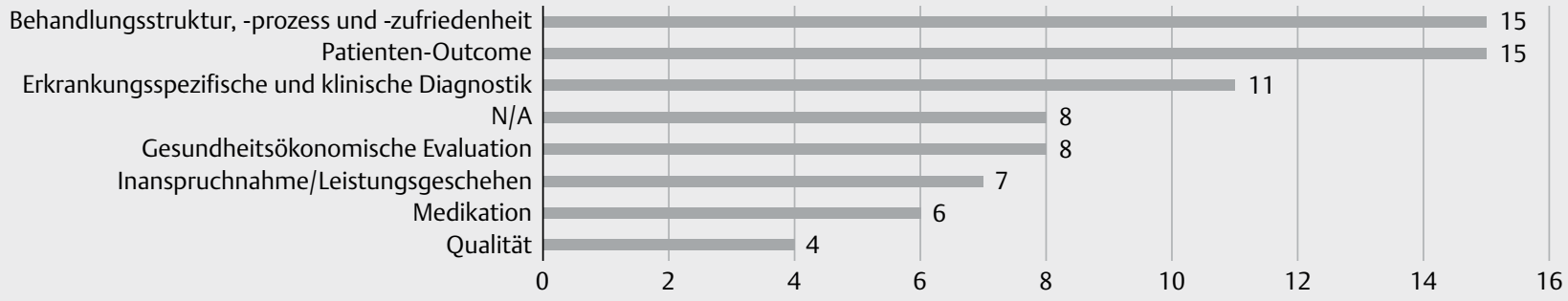

- Abb. 4 Histogramme ausgewählter Charakteristika. ${ }^{*}$ Als „Unspezifiziert“ zusammengefasst sind z. B.: Altersbedingte Komplikationen während und nach einer Operation: Erkrankungen der teilnehmenden Pflegeheim Bewohner; Patienten mit einer unklaren Diagnose oder seltenen Erkrankung.

tiv hochwertige Veröffentlichung unwahrscheinlich, weil (intenationale) Fachjournale ohne Verweis auf ein registriertes Studienprotokoll Manuskripte zu Interventionsstudien praktisch nicht mehr in Betracht ziehen. Eine zentrale Forderung (u. a. des DNVF [12]) nach mehr Transparenz in Form einer verpflichtenden Registrierung öffentlich geförderter Forschungsvorhaben wurde daher nicht ausreichend im Innovationsfonds umgesetzt.
Aus wissenschaftlicher Sicht deutet sich damit die Gefahr nicht ausgeschöpfter Potenziale, ggf. sogar auch die Verschwendung von bereitgestellten Fördermitteln an. Wenn Ergebnisse insbesondere randomisierter aber auch nicht-randomisierter kontrollierter Studien aufgrund mangelhafter oder fehlender Studienregistrierungen an wissenschaftlicher Aussagekraft und Publikationspotenzial stark verlieren, steht die generelle Verwertbarkeit und Transferrelevanz in Frage. 
Wenngleich für eine abschließende Bewertung der Projekte noch Abschlussberichte und Ergebnispublikationen fehlen, zeigt diese Arbeit bereits einen sichtbaren methodischen Verbesserungsund Beratungsbedarf zu zahlreichen gegenwärtigen und zukünftigen Innovationsfonds-Projekten. Das DNVF wird diese qualitativen Untersuchungen zu den weiteren Förderwellen fortsetzen und evidenzgeleitete und methodische Empfehlungen ableiten.

\section{IMitglieder der Ad hoc Kommission Innovationsfonds des Deutschen Netzwerk Versorgungsforschung}

Birga Maier, Jochen Schmitt, Daniel Schwarzkopf, Max Geraedts, Olaf Schoffer, Felix Walther, Christian Apfelbacher, Thomas Bierbaum, Martin Härter, Edmund Neugebauer, Heike Heytens, Felix Mühlensiepen, Laura Keßler, Nicola Reitz, Eva Buchholz, Daniel Bremer, Elisa Frenz, Martin Heinze

\section{Finanzierung}

Eine externe Projektfinanzierung lag nicht vor.

Interessenkonflikt

CA erhielt institutionelle Fördermittel aus dem Innovationsfonds. Außerhalb dieses Projektes erhielt er institutionelle Fördermittel für selbst initiierte Forschungsprojekte durch die Dr. Wolff Group, sowie von der DFG und dem BMBF. Er erhielt Beratungshonorare von Sanofi, LEOPharma und der Dr. Wolff Group, sowie ein Vortragshonorar von AstraZeneca.

DB hat keine Interessenkonflikte.

EF hat keine Interessenkonflikte.

FW war als wissenschaftlicher Mitarbeiter an der in diesem Review mit untersuchten IMPRESS-Studie beteiligt.

$\mathrm{HH}$ hat keine Interessenkonflikte.

JS erhielt institutionelle Fördermittel aus dem Innovationsfonds und ist Hauptgeschäftsführer des DNVF. Außerhalb des Fokus dieses Projekts erhielt er institutionelle Fördermittel für selbst initiierte Forschungsprojekte von Pfizer, Sanofi, Novartis, ALK, sowie von der DFG, vom BMG und vom BMBF. Er war gegen persönliches Honorar als Berater für Sanofi, Lilly und Novartis tätig.

LK hat keine Interessenkonflikte.

MG war als Mitglied des Evaluationsteams an der in diesem Review mit untersuchten INTEGRAL-Studie beteiligt.

MH erhielt institutionelle Fördermittel aus dem Innovationsfonds und ist Vorstandsmitglied im DNVF. Außerhalb dieses Projektes erhielt er institutionelle Fördermittel für selbst initiierte Forschungsprojekte durch das BMG und das BMBF.

TB ist Geschäftsführer des DNVF. Das DNVF hat in der vergangenen Förderwellen beim Innovationsfonds Anträge gestellt, die nicht bewilligt wurden.

\section{Literatur}

[1] Adams O, Guimaraes L, Atherton F, Franzen S. Development Innovation Fund - Health: Summative Evaluation Report. 2015; [cited 2021 Feb 12] Available from: URL https://idl-bnc-idrc.dspacedirect. org/bitstream/handle/10625/55374/IDL-55374.

pdf? sequence $=1$ \& isAllowed $=y$
[2] Bowser D, Sparkes SP, Mitchell A, Bossert T], Bärnighausen T, Gedik C et al. Global Fund investments in human resources for health: innovation and missed opportunities for health systems strengthening. Health policy and planning 2014; 29: 986-997. Available from: URL https://pubmed.ncbi.nlm.nih.gov/24197405/

[3] Lester P. Social Innovation Fund: Early Results Are Promising: Role of Philanthropic Intermediaries, Federal Oversight and Fundingof Evaluations Appear Critical; Regulatory and Matching Requirements Should Be Eased, Knowledge Initiative Expanded. 2015; [cited 2021 Feb 12] Available from: URL http://www.socialinnovationcenter.org/ wp-content/uploads/2015/07/Social_Innovation_Fund-2015-06-30. pdf

[4] Selby JV, Lipstein SH. PCORI at 3 years - progress, lessons, and plans. The New England Journal of Medicine 2014; 370: 592-595

[5] Schmitt ], Geraedts M, Maier B, Schwarzkopf D, Schoffer O, Härter M et al. Zum Status quo und der vorgesehenen Weiterentwicklung des Innovationsfonds (Version 3, 4.2.2020). Gesundheitswesen 2020; 82: 374-377

[6] Schmitt J, Petzold T, Nellessen-Martens G, Pfaff H. Priorisierung und Konsentierung von Begutachtungs-, Förder- und Evaluationskriterien für Projekte aus dem Innovationsfonds: Eine multiperspektivische Delphi-Studie. Gesundheitswesen 2015; 77: 570-579

[7] WHO Terminology Information System: Online glossary. Intervention; o. J. [cited 2020 Nov 18] Available from: URL http://www.who.int/ health-systems-performance/docs/glossary.htm 2020-05-11.

[8] Deutsches Register Klinischer Studien. Glossary of key terms: Study t ype interventional; o. J. [cited 2021 Feb 11] Available from: URL https:// www.drks.de/drks_web/navigate.do?navigationld = glossar \&messageDE $=$ Glossar\&messageEN $=$ Glossary\#Intervention. \%20 2020-05-11

[9] Deutscher Bundestag. Zwischenbericht über die wissenschaftliche Auswertung der Förderung durch den Innovationsfonds im Hinblick auf deren Eignung zur Weiterentwicklung der Versorgung: Drucksache 19/8500; 2019 [cited 2021 Feb 28] Available from: URL https://dipbt. bundestag.de/doc/btd/19/085/1908500.pdf

[10] Deutscher Bundestag. Gesamtevaluation des Innovationsfonds: Teilbericht über die erste Evaluationsphase; 2019 [cited 2021 Mar 19] Available from: URL https://www.prognos.com/de/projekt/gesamtevaluation-des-innovationsfonds

[11] Greß S, Schnee M. Wege zur integrierten und sektorenübergreifenden Versorgung. G+G Wissenschaft (GGW). 2017; 17: 7-15.Available from: URL https://www.wido.de/fileadmin/Dateien/Dokumente/ Publikationen_Produkte/GGW/wido_ggw_0317_gress_schnee.pdf

[12] Nellessen-Martens G, Hoffman W. Versorgungsforschung - eine Disziplin im Aufschwung. G + G Wissenschaft (GGW) 2017; 17: 7-15. Available from: URL https://www.wido.de/fileadmin/Dateien/ Dokumente/Publikationen_Produkte/GGW/wido_ggw_0117_nellessen_hoffmann.pdf

\section{Weitere Literatur}

Adrion C, Weiss B, Paul N et al. Enhanced Recovery after Intensive Care (ERIC): study protocol for a German stepped wedge cluster randomised controlled trial to evaluate the effectiveness of a critical care telehealth program on process quality and functional outcomes. BMJ Open 2020; 10: 9

Altiner A. Reducing antibiotic resistance through adequate use of antibiotics. 2018; Im Internet: https://www.isrctn.com/ ISRCTN13934505?q = \& filters = acronym:RESIST\&sort = \&offset = 1\&totalResults $=2 \&$ page $=1 \&$ pageSize $=10 \&$ searchType $=$ advanced-search; Stand: 15.20.2021].

Andres E, Kaufmann-Kolle P, Wambach $V$ et al. Antibiotika-Resistenzentwicklung nachhaltig abwenden (ARena): Erste Ergebnisse zur Verbesserung der hausärztlichen Versorgung; 2019 
AOK Baden-Württemberg. Schlussbericht: Vertragsevaluation der Fachgebiete Neurologie, Psychiatrie, Psychosomatik und Psychotherapie in Baden-Württemberg gem. § 73 SGB V; 2020. Im Internet: https://neueversorgung.de/images/PDF/20200630_Schlussbericht_ PNP_Evaluation.pdf; Stand: 08.02.2021].

Ärzte Zeitung. TELnet@NRW. Expertise aus Unikliniken für Arztpraxen 2020; Im Internet: https://www.aerztezeitung.de/Wirtschaft/ Expertise-aus-Unikliniken-fuer-Arztpraxen-405902.html; Stand: 08.02.2021]

Audebert H. ANNOTeM (Akut-Neurologische Versorgung in Nord-Ost-Deutschland mit Telemedizinischer Unterstützung). Effekte der telemedizinisch unterstützten Notfallversorgung akutneurologischer Krankheitsbilder; 2017. Im Internet: https://www.drks.de/ drks_web/navigate.do?navigationld = trial.HTML\&TRIAL _ ID = DRKS00013067; Stand: 15.02.2021]

Austenat-Wied M. Allgemein-, Fach- und Notfallmedizin im ländlichen Raum am Beispiel Dermatologie; 2018

Bajbouj M. Mental Health in Refugees and Asylum Seekers (MEHIRA). 2017; Im Internet: https://clinicaltrials.gov/ct2/show/NCT03109028? term $=$ MEHIRA\&cntry $=$ DE\&draw $=2 \&$ rank $=1$ Stand: 15.02 .2021$]$.

Bergelt $C$. Entwicklung und Erprobung eines komplexen interprofessionellen Trainingsprogramms zur Verbesserung der Patientensicherheit (KOMPAS). 2017; Im Internet: https://www.drks.de/drks_web/ navigate.do? navigationld $=$ trial. $\cdot$ HTML\&TRIAL_ID = DRKS00012818 Stand: 15.02.2021].

Berndt R-D, Preik P, Takenga C. TeleDermatologie. Der Hautarzt 2019; 70 (5): 335-42.

Betz U, Langanki L, Heid F et al. The PROMISE study protocol: a multicenter prospective study of process optimization with interdisciplinary and cross-sectoral care for German patients receiving hip and knee endoprostheses. Acta Orthopaedica 2020; doi:10.1080/17453 674.2020.1853927

Bock de F, Neubert A, Binder $\mathrm{H}$ et al. Evaluation einer komplexen Intervention zur Erhöhung der Arzneimitteltherapiesicherheit bei Kindern in der ambulanten und stationären Versorgung. 2017;

Boer de S, Klewitz F, Bauer-Hohmann M et al. Knowledge About Immunosuppressant Medication and Its Correlates in a German Kidney Transplant Population - Results of a KT× $360^{\circ}$ Substudy. Patient Prefer Adherence 2020; 14: 1699-708

Böge K, Karnouk C, Hahn E et al. Mental health in refugees and asylum seekers (MEHIRA): study design and methodology of a prospective multicentre randomized controlled trail investigating the effects of a stepped and collaborative care model. European Archives of Psychiatry and Clinical Neuroscience 2020; 270 (1): 95-106

Bothe P, Pfaff H, Lindert L. BGM-innovativ: Arbeitsplatznahes, trägerübergreifendes Versorgungsmanagement der Betriebskrankenkassen. 2017;

Brandl M, Weiß A, Bernardi C et al. PINA: Perspektiven von Versorgungsakteuren zur Entwicklung einer Intensiv-Nachsorgeambulanz. 2019 Im Internet: https://www.egms.de/static/en/meetings/ dkvf2019/19dkvf203.shtm

Brandstetter S, Baumeister S, Pfeifer M et al. Folgeschäden nach prolongierter Intensivbehandlung: Entwicklung und Pilotierung einer Intensiv-Nachsorge-Ambulanz (PINA); 2017

Brinkrolf P., Scheer D., Hasebrook J., Hahnenkamp K. Land|Rettung: Zukunftsfeste notfallmedizinische Neuausrichtung eines Landkreises. 2017; Im Internet" https://www.researchgate.net/publication/314511727_LandRettung_Zukunftsfeste_notfallmedizinische_ Neuausrichtung_eines_Landkreises Stand: 19.03.21].

Brühmann B, Reese C, Kaier $\mathrm{K}$ et al. A complex health services intervention to improve medical care in long-term care homes: study protocol of the controlled coordinated medical care (CoCare) study. BMC Health Services Research 2019; 19 (1): 332
ClinicalTrials.gov. Improvement of Perioperative Care of Elderly Patients (PeriAge); 2017; Stand: 15.02.2021]. Im Internet: https:// clinicaltrials.gov/ct2/show/NCT03325413?term $=$ PeriAge \&cntry $=$ DE $\&$ draw $=2 \&$ rank $=1$

ClinicalTrials.gov. ICU Follow-up After Prolonged Intensive Care Stay (PINA); 2019; Stand: 15.02.2021.] Im Internet: https://clinicaltrials. gov/ct2/show/NCT04186468?term $=$ PINA\&cntry $=$ DE\&draw $=2 \&$ rank $=1$

Cochrane Central Register of Controlled Trials (CENTRAL). Process optimization by interdisciplinary and cross-sectoral care using the example of patients with hip and knee prostheses 2019; Im Internet: https://www.cochranelibrary.com/central/doi/10.1002/central/ CN-01898218/full.

Decke S, Deckert K, Lang M et al. "We're in good hands there." - Acceptance, barriers and facilitators of a primary care-based health coaching programme for children and adolescents with mental health problems: a qualitative study (PrimA-QuO). BMC Fam Pract 2020; 21 (1): 273

Deeken F, Thomas C, Eschweiler G et al. Die PAWEL-Studie - Prävention von Delir und kognitiver Dysfunktion nach Elektivoperationen im Alter; 2019

Dinger $\mathrm{U}$, Komo-Lang M, Brunner $\mathrm{F}$ et al. Neue Behandlungsformen. Psychotherapeut 2016; 61 (2): 141-7

Dinger $\mathrm{U}$, Komo-Lang M, Schauenburg $\mathrm{H}$ et al. Die psychotherapeutische Abendklinik: Konzept und erste Ergebnisse. Psychother Psychosom Med Psychol 2019; 69 (5): 197-202

Dinius J, Heier L, Pfisterer-Heise $S$ et al. Blended Learning für interprofessionelle Teams zur Verbesserung der Patientensicherheit in der stationären akutmedizinischen Versorgung. Gesundheitswesen 2019; 81 (8/9): 4I-10.

Dinius J, Hammer A, Manser T et al. Piloting and evaluating feasibility of a training program to improve patient safety for inter-professional inpatient care teams - study protocol of a cluster randomized controlled trial. Trials 2019; 20 (1): 386

Dinius J, Philipp R, Ernstmann $\mathrm{N}$ et al. Inter-professional teamwork and its association with patient safety in German hospitals-A cross sectional study. PLoS One 2020; 15: 5

Dohmen S, Benstoem C, Wahl A et al. Qualitätssteigerung in der Intensivmedizin durch Telemedizin: Beispiel TELnet@NRW. Anästhesiol Intensivmed Notfallmed Schmerzther 2021; 56 (1): 52-9

Done N, Oh D, Weinstock M et al. VA Telederm study: protocol for a stepped-wedge cluster randomised trial to compare access to care for a mobile app versus a workstation-based store-and-forward teledermatology process. BMJ Open 2018; 8: 12

Döpfner M.. Evaluation eines web-assistierten Selbsthilfe-Trainings für Eltern von Kindern mit Aufmerksamkeitsdefizit- / Hyperaktivitätsstörung. 2017; Im Internet: https://www.drks.de/drks_web/navigate. do? navigationld = trial.HTML\&TRIAL_ID = DRKS00013456; Stand: 15.02.2021].

Döpfner M, Wähnke L, Klemp M-T et al. Efficacy of web-assisted self-help for parents of children with ADHD (WASH) - a three-arm randomized trial under field/routine care conditions in Germany. BMC Psychiatry 2020; 20 (1): 76

Doyle I-M, Kirsch C, Thürmann P et al. Studienprotokoll einer Cluster-randomisierten Studie zur Erforschung der angemessenen und sicheren Medikation bei Heimbewohnern mithilfe einer interprofessionellen Toolbox; 2017

Eschweiler G. PAWEL: Patientensicherheit, Wirtschaftlichkeit und Lebensqualität: Reduktion von Delirrisiko und POCD nach Elektivoperationen im Alter. 2017; Im Internet: https://www.drks.de/drks_web/ navigate.do?navigationld $=$ trial.HTML\&TRIAL_ID = DRKS00013311 Stand: 15.02 .2021$]$. 
Farin-Glattacker E, Kucher R, Kunert S et al. Implementierung und Evaluation einer Intervention zur Verbesserung der ärztlichen Versorgung in Pflegeheimen - Studienprotokoll des Projekts CoCare (Coordinated. Medical Care); 2017;

Fichtner U, Arslanow A, Beyer A et al. Psychosoziale Aspekte eines Lebervorsorgescreenings in Rheinland-Pfalz und im Saarland; 2020

Förstermann U.. Prozessoptimierung durch interdisziplinäre und sektorenübergreifende Versorgung am Beispiel von Patienten mit Hüft- und Knieendoprothesen. 2018; Im Internet: https://www.drks. de/drks_web/navigate.do?navigationld = trial.HTML\&TRIAL_ ID = DRKS00013972; Stand: 15.02.2021].

Förstermann U. SEAL - Strukturierte Früherkennung einer asymptomatischen Leberfibrose und Leberzirrhose. 2018; Im Internet: https:// www.drks.de/drks_web/navigate.do?navigationld = trial. HTML\&TRIAL_ID = DRKS00013460; Stand: 15.02.2021].

Freiberg L.. IGiB - Strukturmigration im Mittelbereich Templin (StimMT): zukunftsfähige Gestaltung regionaler Versorgung. G\&S Gesundheits- und Sozialpolitik 2017; 71 (1): 35-9

Frielitz F-S, Dördelmann J, Lemke $S$ et al. Assessing the benefits and challenges of video consultations for the treatment of children with type 1 diabetes - A qualitative study among diabetes professionals. Exp Clin Endocrinol Diabetes. 2020; (EFirst)

Frielitz F-S, Müller-Godeffroy E, Hübner J et al. Monthly Video-Consultation for Children With Type 1 Diabetes Using a Continuous Glucose Monitoring System: Design of ViDiKi, a Multimethod Intervention Study to Evaluate the Benefit of Telemedicine. J Diabetes Sci Technol 2020; 14 (1): 105-11.

Fuchs A, Mortsiefer A, Wilm S et al. Change Management als Intervention zur Verbesserung der Arzneimitteltherapiesicherheit in Pflegeheimen (HIOPP-3-iTBX-Studie) 2018

Gavenis K.. Effect of Strategies to Improve General Practitioner-nurse Collaboration and Communication (interprof ACT). 2018; Im Internet: https://clinicaltrials.gov/ct2/show/NCT03426475?term = interprof + ACT\&cntry = DE\&draw $=2 \&$ rank $=1$; Stand: 15.02 .2021$]$.

Geissler A, Berger E.. Enhanced Recovery after Intensive Care - ERIC; 2017

Gemeinsamer Bundesausschuss G-BA - Innovationsausschuss. ACD - Accountable Care in Deutschland - Verbesserung der Patientenversorgung durch Vernetzung von Leistungserbringern und informierten Dialog: Projektbeschreibung. 2021;Stand: 21.01.2021]. Im Internet: https:// innovationsfonds.g-ba.de/projekte/versorgungsforschung/acd-accountable-care-in-deutschland-verbesserung-der-patientenversorgung-durchvernetzung-von-leistungserbringern-und-informierten-dialog. 45

Gemeinsamer Bundesausschuss G-BA - Innovationsausschuss. AdAM

- Anwendung digital-gestütztes Arzneimitteltherapie- und

Versorgungs-Management: Projektbeschreibung 2021; Stand: 21.01.2021]. Im Internet: https://innovationsfonds.g-ba.de/projekte/ neue-versorgungsformen/adam-anwendung-digital-gestuetztesarzneimitteltherapie-und-versorgungs-management.71

Gemeinsamer Bundesausschuss G-BA - Innovationsausschuss. AHeaD - Künftige Aufgabenteilung von Pflegefachpersonen und Hausärzten in der ambulanten Demenzversorgung: Aufgaben, Akzeptanz, Qualifikation: Projektbeschreibung 2021; Stand: 21.01.2021]. Im Internet: https://innovationsfonds.g-ba.de/projekte/versorgungsforschung/ahead-kuenftige-aufgabenteilung-von-pflegefachpersonenund-hausaerzten-in-der-ambulanten-demenzversorgung-aufgabenakzeptanz-qualifikation.28

Gemeinsamer Bundesausschuss G-BA - Innovationsausschuss. AMTS in utero - Untersuchungen zur Arzneimitteltherapiesicherheit in der Schwangerschaft basierend auf Routinedaten in Deutschland: Projektbeschreibung 2021; Stand: 21.01.2021]. Im Internet: https:// innovationsfonds.g-ba.de/projekte/versorgungsforschung/amts-inutero-untersuchungen-zur-arzneimitteltherapiesicherheit-in-derschwangerschaft-basierend-auf-routinedaten-in-deutschland.10
Gemeinsamer Bundesausschuss G-BA - Innovationsausschuss. ANNOTeM - Akut-Neurologische Versorgung in Nord- Ost-Deutschland mit TeleMedizinischer Unterstützung: Projektbeschreibung 2021; Stand: 21.01.2021]. Im Internet: https:// innovationsfonds.g-ba.de/projekte/neue-versorgungsformen/ annotem-akut-neurologische-versorgung-in-nord-ost-deutschlandmit-telemedizinischer-unterstuetzung.76

Gemeinsamer Bundesausschuss G-BA - Innovationsausschuss. APVEL - Evaluation der Wirksamkeit von SAPV in Nordrhein: Projektbeschreibung 2021;Stand: 21.01.2021]. Im Internet: https:// innovationsfonds.g-ba.de/projekte/versorgungsforschung/ apvel-evaluation-der-wirksamkeit-von-sapv-in-nordrhein.7

Gemeinsamer Bundesausschuss G-BA - Innovationsausschuss. ARena - Antibiotika-Resistenzentwicklung nachhaltig abwenden: Projektbeschreibung 2021;Stand: 21.01.2021] Im Internet https:// innovationsfonds.g-ba.de/projekte/neue-versorgungsformen/ arena-antibiotika-resistenzentwicklung-nachhaltig-abwenden.86

Gemeinsamer Bundesausschuss G-BA - Innovationsausschuss. BGM-innovativ - Arbeitsplatznahes, trägerübergreifendes Versorgungsmanagement der Betriebskrankenkassen: Projektbeschreibung 2021;Stand: 21.01.2021] Im Internet https://innovationsfonds.g-ba. de/projekte/neue-versorgungsformen/bgm-innovativ-arbeitsplatznahes-traegeruebergreifendes-versorgungsmanagement-der-betriebskrankenkassen. 75

Gemeinsamer Bundesausschuss G-BA - Innovationsausschuss. CIRSforte - Projekt zur Fortentwicklung von Fehlerberichts- und Lernsystemen (CIRS) für die ambulante Versorgung zu einem implementierungsreifen System: Projektbeschreibung 2021; Stand: 21.01.2021]. Im Internet: https://innovationsfonds.g-ba.de/projekte/ versorgungsforschung/cirsforte-projekt-zur-fortentwicklung-vonfehlerberichts-und-lernsystemen-cirs-fuer-die-ambulante-versorgung-zu-einem-implementierungsreifen-system.21

Gemeinsamer Bundesausschuss G-BA - Innovationsausschuss. CoCare (coordinated medical care) - Erweiterte koordinierte ärztliche Pflegeheimversorgung: Projektbeschreibung 2021; Stand: 21.01.2021]. Im Internet: https://innovationsfonds.g-ba.de/projekte/ neue-versorgungsformen/cocare-coordinated-medical-care-erweiterte-koordinierte-aerztliche-pflegeheimversorgung.70

Gemeinsamer Bundesausschuss G-BA - Innovationsausschuss. DELIVER - Determinanten für leitlinieninkongruente Versorgung von älteren Krebspatienten in der GKV: Projektbeschreibung 2021; Stand: 21.01.2021]. Im Internet: https://innovationsfonds.g-ba.de/projekte/ versorgungsforschung/deliver-determinanten-fuer-leitlinieninkongruente-versorgung-von-aelteren-krebspatienten-in-der-gkv.34

Gemeinsamer Bundesausschuss G-BA - Innovationsausschuss. Dent@ Prevent - Implementierung von Routinedaten \& PROMS in die evidenz-informierte intersektorale (zahn-)medizinische Versorgung: Projektbeschreibung 2021; Stand: 21.01.2021]. Im Internet: https:// innovationsfonds.g-ba.de/projekte/versorgungsforschung/ dentatprevent-implementierung-von-routinedaten-proms-in-dieevidenz-informierte-intersektorale-zahn-medizinische-versorgung.51

Gemeinsamer Bundesausschuss G-BA - Innovationsausschuss. DEWI - Determinanten bei der Versorgung von Patienten mit Wirbelsäulenoperation: Projektbeschreibung 2021; Stand: 21.01.2021], Im Internet: https://innovationsfonds.g-ba.de/projekte/versorgungsforschung/dewi-determinanten-bei-der-versorgung-von-patienten-mitwirbelsaeulenoperation. 44

Gemeinsamer Bundesausschuss G-BA - Innovationsausschuss. ELSAH - Evaluation der Spezialisierten Ambulanten Palliativversorgung (SAPV) am Beispiel von Hessen: Projektbeschreibung 2021; Stand: 21.01.2021]. Im Internet: https://innovationsfonds.g-ba.de/projekte/ versorgungsforschung/elsah-evaluation-der-spezialisierten-ambulanten-palliativversorgung-sapv-am-beispiel-von-hessen. 6 
Gemeinsamer Bundesausschuss G-BA - Innovationsausschuss. EMPAR - Einfluss metabolischer Profile auf die Arzneimitteltherapiesicherheit in der Routineversorgung: Projektbeschreibung 2021; Stand: 21.01.2021]. Im Internet: https://innovationsfonds.g-ba.de/projekte/ versorgungsforschung/empar-einfluss-metabolischer-profile-auf-diearzneimitteltherapiesicherheit-in-der-routineversorgung.46

Gemeinsamer Bundesausschuss G-BA - Innovationsausschuss. EMSE - Entwicklung von Methoden zur Nutzung von Routinedaten für ein Sektorenübergreifendes Entlassmanagement: Projektbeschreibung 2021; Stand: 21.01.2021]. Im Internet: https:// innovationsfonds.g-ba.de/projekte/versorgungsforschung/ emse-entwicklung-von-methoden-zur-nutzung-von-routinedatenfuer-ein-sektorenuebergreifendes-entlassmanagement.40

Gemeinsamer Bundesausschuss G-BA - Innovationsausschuss. EPOS - Ergebnisqualität durch Patient Reported Outcome Measures (PROMs) bei Schlaganfallpatienten in der klinischen Routine: Projektbeschreibung 2021; Stand: 21.01.2021] .Im Internet: https:// innovationsfonds.g-ba.de/projekte/versorgungsforschung/ epos-ergebnisqualitaet-durch-patient-reported-outcome-measuresproms-bei-schlaganfallpatienten-in-der-klinischen-routine.23

Gemeinsamer Bundesausschuss G-BA - Innovationsausschuss. ERIC - Enhanced Recovery after Intensive Care: Projektbeschreibung 2021; Stand: 21.01.2021]. Im Internet :https:// innovationsfonds.g-ba.de/projekte/neue-versorgungsformen/ eric-enhanced-recovery-after-intensive-care.66

Gemeinsamer Bundesausschuss G-BA - Innovationsausschuss. Evaluation Kardiologie-Vertrag - Evaluation des Vertrages zur Versorgung im Fachgebiet der Kardiologie in Baden-Württemberg gemäß § 73 c SGB V (Kardiologie-Vertrag): Projektbeschreibung 2021; Stand: 21.01.2021]. Im Internet: https://innovationsfonds.g-ba.de/ projekte/versorgungsforschung/evaluation-des-vertrages-zur-versorgung-im-fachgebiet-der-kardiologie-in-baden-wuerttemberg-gemaess-73-c-sgb-v-kardiologie-vertrag.3

Gemeinsamer Bundesausschuss G-BA - Innovationsausschuss. Evaluation PNP-Vertrag - Vertragsevaluation der Fachgebiete Neurologie, Psychiatrie, Psychosomatik und Psychotherapie in Baden-Württemberg gem. § 73c SGB V: Projektbeschreibung 2021; Stand: 21.01.2021]. Im Internet: https://innovationsfonds.g-ba.de/ projekte/versorgungsforschung/vertragsevaluation-der-fachgebieteneurologie-psychiatrie-psychosomatik-und-psychotherapie-inbaden-wuerttemberg-gem-73c-sgb-v. 1

Gemeinsamer Bundesausschuss G-BA - Innovationsausschuss. EVITA - Evidenzbasiertes Multimedikations-Programm mit Implementierung in die Versorgungspraxis: Projektbeschreibung 2021; Stand: 21.01.2021]. Im Internet: https://innovationsfonds.g-ba.de/projekte/ versorgungsforschung/evita-evidenzbasiertes-multimedikationsprogramm-mit-implementierung-in-die-versorgungspraxis.33

Gemeinsamer Bundesausschuss G-BA - Innovationsausschuss. EyeLLIS - Eyetracking-basierte Erhebung der Lebensqualität von Patienten mit Locked-in-Syndrom: Projektbeschreibung 2021; Stand: 21.01.2021]. Im Internet: https://innovationsfonds.g-ba.de/projekte/ versorgungsforschung/eyellis-eyetracking-basierte-erhebung-derlebensqualitaet-von-patienten-mit-locked-in-syndrom.26

Gemeinsamer Bundesausschuss G-BA - Innovationsausschuss. Frauen 5.0 - Regionale Versorgung von Frauen über 49 Jahren durch Fachärzte und Fachärztinnen für Gynäkologie und für Allgemeinmedizin: Projektbeschreibung 2021; Stand: 21.01.2021]. Im Internet: https://innovationsfonds.g-ba.de/projekte/versorgungsforschung/ frauen-5-0-regionale-versorgung-von-frauen-ueber-49-jahrendurch-fachaerzte-und-fachaerztinnen-fuer-gynaekologie-und-fuerallgemeinmedizin. 29

Gemeinsamer Bundesausschuss G-BA - Innovationsausschuss. HaReNa - Hausarztzentrierte Reha-Nachsorge bei Rückenschmerzen (RS): Projektbeschreibung 2021; Stand: 21.01.2021]. Im Internet: https:// innovationsfonds.g-ba.de/projekte/versorgungsforschung/harenahausarztzentrierte-reha-nachsorge-bei-rueckenschmerzen-rs.53
Gemeinsamer Bundesausschuss G-BA - Innovationsausschuss. Heimeintritt vermeiden - Beginn stationärer Langzeitpflege und seine Prädiktoren in der Versorgungs-, Wohn- und Unterstützungssituation - populationsbasierte Kohortenstudie: Projektbeschreibung 2021; Stand: 21.01.2021] .Im Internet: https:// innovationsfonds.g-ba.de/projekte/versorgungsforschung/ heimeintritt-vermeiden-beginn-stationaerer-langzeitpflege-undseine-praediktoren-in-der-versorgungs-wohn-und-unterstuetzungssituation-populationsbasierte-kohortenstudie.41

Gemeinsamer Bundesausschuss G-BA - Innovationsausschuss. HELP@ APP - Entwicklung und Evaluation einer Selbsthilfe-App für traumatisierte syrische Flüchtlinge in Deutschland: Projektbeschreibung 2021; Stand: 21.01.2021]. Im Internet: https:// innovationsfonds.g-ba.de/projekte/versorgungsforschung/ helpatapp-entwicklung-und-evaluation-einer-selbsthilfe-app-fuertraumatisierte-syrische-fluechtlinge-in-deutschland.32

Gemeinsamer Bundesausschuss G-BA - Innovationsausschuss. HerzEffekt MV - Entwicklung und spezifischer Aufbau eines sektorenübergreifenden Care-Centers zur Versorgungsoptimierung chronischer Herzerkrankungen in MV: Projektbeschreibung 2021; Stand: 21.01.2021]. Im Internet: https://innovationsfonds.g-ba.de/ projekte/neue-versorgungsformen/herzeffekt-mv-entwicklung-undspezifischer-aufbau-eines-sektorenuebergreifenden-care-centers-zurversorgungsoptimierung-chronischer-herzerkrankungen-in-mv.82

Gemeinsamer Bundesausschuss G-BA - Innovationsausschuss. HIOPP-3-iTBX - Angemessene und sichere Medikation für Heimbewohner/innen mit Hilfe einer interprofessionellen Toolbox (AMTS-Toolbox): Projektbeschreibung 2021; Stand: 21.01.2021]. Im Internet: https:// innovationsfonds.g-ba.de/projekte/versorgungsforschung/hiopp-3-itbxangemessene-und-sichere-medikation-fuer-heimbewohner-innen-mithilfe-einer-interprofessionellen-toolbox-amts-toolbox.17

Gemeinsamer Bundesausschuss G-BA - Innovationsausschuss. HIOPP-6 - Projekt aus dem HIOPP Forschungsverbund: Hausärztliche Initiative zur Optimierung der Patientensicherheit bei Polypharmazie - Komplexitätsreduktion in der Polypharmazie unter Beachtung von Patientenpräferenzen: Projektbeschreibung 2021; Stand: 21.01.2021]. Im Internet: https://innovationsfonds.g-ba.de/projekte/ versorgungsforschung/hiopp-6-projekt-aus-dem-hiopp-forschungsverbund-hausaerztliche-initiative-zur-optimierung-der-patientensicherheit-bei-polypharmazie-komplexitaetsreduktion-in-der-polypharmazie-unter-beachtung-von-patientenpraeferenzen.19

Gemeinsamer Bundesausschuss G-BA - Innovationsausschuss. Homern - Hospitalisierung und Notaufnahmebesuche von Pflegeheimbewohnern: Häufigkeit, Ursachen und Entwicklung einer Intervention zur Verbesserung der Versorgung: Projektbeschreibung 2021;Stand: 21.01.2021]. Im Internet: https://innovationsfonds.g-ba. de/projekte/versorgungsforschung/homern-hospitalisierung-undnotaufnahmebesuche-von-pflegeheimbewohnern-haeufigkeit-ursachen-und-entwicklung-einer-intervention-zur-verbesserung-derversorgung. 54

Gemeinsamer Bundesausschuss G-BA - Innovationsausschuss. IDOMENEO Studie - Ist die Versorgungsrealität in der Gefäßmedizin leitlinien- und versorgungsgerecht: Projektbeschreibung 2021; Stand: 21.01.2021]. Im Internet: https://innovationsfonds.g-ba.de/projekte/ versorgungsforschung/idomeneo-studie-ist-die-versorgungsrealitaetin-der-gefaessmedizin-leitlinien-und-versorgungsgerecht.8

Gemeinsamer Bundesausschuss G-BA - Innovationsausschuss. IGiB-StimMT - Strukturmigration im Mittelbereich Templin: Projektbeschreibung 2021; Stand: 21.01.2021]. Im Internet: https:// innovationsfonds.g-ba.de/projekte/neue-versorgungsformen/ igib-stimmt-strukturmigration-im-mittelbereich-templin.79 
Gemeinsamer Bundesausschuss G-BA - Innovationsausschuss. IMPRESS - Effektivität des IQM-Peer Review Verfahrens zur Verbesserung der Ergebnisqualität - eine pragmatische clusterrandomisierte kontrollierte Studie: Projektbeschreibung 2021; Stand: 21.01.2021]. Im Internet: https://innovationsfonds.g-ba.de/ projekte/versorgungsforschung/impress-effektivitaet-des-iqm-peerreview-verfahrens-zur-verbesserung-der-ergebnisqualitaet-einepragmatische-cluster-randomisierte-kontrollierte-studie.13

Gemeinsamer Bundesausschuss G-BA - Innovationsausschuss. INDEED - Inanspruchnahme und sektorenübergreifende Versorgungsmuster von Patienten in Notfallversorgungsstrukturen in Deutschland: Projektbeschreibung 2021; Stand: 21.01.2021]. Im Internet: https://innovationsfonds.g-ba.de/projekte/versorgungsforschung/indeed-inanspruchnahme-und-sektorenuebergreifendeversorgungsmuster-von-patienten-in-notfallversorgungsstrukturenin-deutschland.43

Gemeinsamer Bundesausschuss G-BA - Innovationsausschuss. INSIST - Impact des Neonatologie-Screenings auf Infektionsprädiktion / Senkung von Transmission: Projektbeschreibung 2021; Stand: 21.01.2021]. Im Internet: https://innovationsfonds.g-ba.de/projekte/ versorgungsforschung/insist-impact-des-neonatologie-screeningsauf-infektionspraediktion-senkung-von-transmission.50

Gemeinsamer Bundesausschuss G-BA - Innovationsausschuss. INTEGRAL - 10-jahres-Evaluation der populationsbezogenen integrierten Versorgung Gesundes Kinzigtal in Aufbau- und Konsolidierungsphase: Projektbeschreibung 2021; Stand: 21.01.2021. Im Internet: INTEGRAL - 10-Jahres-Evaluation der populationsbezogenen integrierten Versorgung Gesundes Kinzigtal in Aufbau- und Konsolidierungsphase

Gemeinsamer Bundesausschuss G-BA - Innovationsausschuss. interprof ACT - Effekte von Strategien zur Verbesserung ärztlichpflegerischer Zusammenarbeit auf Krankenhausaufnahmen von Pflegeheimbewohnern: Projektbeschreibung 2021; Stand: 21.01.2021]. Im Internet: https://innovationsfonds.g-ba.de/projekte/ versorgungsforschung/interprof-act-effekte-von-strategien-zurverbesserung-aerztlich-pflegerischer-zusammenarbeit-auf-krankenhausaufnahmen-von-pflegeheimbewohnern.62

Gemeinsamer Bundesausschuss G-BA - Innovationsausschuss. INVEST Billstedt/Horn - Hamburg Billstedt/Horn als Prototyp für eine Integrierte gesundheitliche Vollversorgung in deprivierten großstädtischen Regionen: Projektbeschreibung 2021; Stand: 21.01.2021]. Im Internet: https://innovationsfonds.g-ba.de/projekte/ neue-versorgungsformen/invest-billstedt-horn-hamburg-billstedthorn-als-prototyp-fuer-eine-integrierte-gesundheitliche-vollversorgung-in-deprivierten-grossstaedtischen-regionen.73

Gemeinsamer Bundesausschuss G-BA - Innovationsausschuss. IpKiSuN - Unterstützende Intensivprophylaxe für Kinder mit zahnärztlicher Sanierung unter Narkose: Projektbeschreibung 2021; Stand: 21.01.2021]. Im Internet: https://innovationsfonds.g-ba.de/projekte/ neue-versorgungsformen/ipkisun-unterstuetzende-intensivprophylaxe-fuer-kinder-mit-zahnaerztlicher-sanierung-unter-narkose.88

Gemeinsamer Bundesausschuss G-BA - Innovationsausschuss. IVF2F3 - Integrierter Versorgungsvertrag Schizophrenie und Depression: Projektbeschreibung 2021; Stand: 21.01.2021]. Im Internet: https:// innovationsfonds.g-ba.de/projekte/versorgungsforschung/ivf2f3integrierter-versorgungsvertrag-schizophrenie-und-depression.4

Gemeinsamer Bundesausschuss G-BA - Innovationsausschuss. KARDIO-Studie - Linksherzkatheter bei Brustschmerzen und KHK: Analyse regionaler Variationen und Behandlungspfade zur Verbesserung der Indikationsqualität: Projektbeschreibung 2021;Stand: 21.01.2021]. Im Internet: https://innovationsfonds.g-ba.de/projekte/ versorgungsforschung/kardio-linksherzkatheter-bei-brustschmerzenund-khk-analyse-regionaler-variationen-und-behandlungspfade-zurverbesserung-der-indikationsqualitaet.47
Gemeinsamer Bundesausschuss G-BA - Innovationsausschuss. KiDSafe - Verbesserung der Versorgung von Kindern und Jugendlichen mit Arzneimitteln durch Erhöhung der Arzneimitteltherapiesicherheit: Projektbeschreibung 2021; Stand: 21.01.2021]. Im Internet: https://innovationsfonds.g-ba.de/projekte/neue-versorgungsformen/kidsafe-verbesserung-der-versorgung-von-kindernund-jugendlichen-mit-arzneimitteln-durch-erhoehung-der-arzneimitteltherapiesicherheit.81

Gemeinsamer Bundesausschuss G-BA - Innovationsausschuss. KOL-OPT_UH - Fehlversorgung bzgl. Kontroll-Koloskopien in Deutschland: Ausmaß, Determinanten und Konzipierung von Lösungsansätzen: Projektbeschreibung 2021; Stand: 21.01.2021]. Im Internet: https:// innovationsfonds.g-ba.de/projekte/versorgungsforschung/kol-opt-uhfehlversorgung-bzgl-kontroll-koloskopien-in-deutschland-ausmassdeterminanten-und-konzipierung-von-loesungsansaetzen.36

Gemeinsamer Bundesausschuss G-BA - Innovationsausschuss. KOMPAS - Entwicklung und Erprobung eines komplexen interprofessionellen Trainingsprogramms zur Verbesserung der Patientensicherheit: Projektbeschreibung 2021; Stand: 21.01.2021]. Im Internet: https:// innovationsfonds.g-ba.de/projekte/versorgungsforschung/kompasentwicklung-und-erprobung-eines-komplexen-interprofessionellentrainingsprogramms-zur-verbesserung-der-patientensicherheit.14

Gemeinsamer Bundesausschuss G-BA - Innovationsausschuss. LandRettung - Zukunftsfeste notfallmedizinische Neuausrichtung eines Landkreises: Projektbeschreibung 2021; Stand: 21.01.2021]. Im Internet: https://innovationsfonds.g-ba.de/projekte/neue-versorgungsformen/landrettung-zukunftsfeste-notfallmedizinische-neuausrichtung-eines-landkreises.63

Gemeinsamer Bundesausschuss G-BA - Innovationsausschuss. LQ-DMP - Lebensqualität im Disease Management Programm COPD: Projektbeschreibung 2021; Stand: 21.01.2021]. Im Internet: https:// innovationsfonds.g-ba.de/projekte/versorgungsforschung/lq-dmplebensqualitaet-im-disease-management-programm-copd.25

Gemeinsamer Bundesausschuss G-BA - Innovationsausschuss. MAU-PD - Multidimensionale Analyse der Ursachen für die niedrige Prävalenz der ambulanten Peritonealdialyse in Deutschland: Projektbeschreibung 2021; Stand: 21.01.2021]. Im Internet: https:// innovationsfonds.g-ba.de/projekte/versorgungsforschung/ mau-pd-multidimensionale-analyse-der-ursachen-fuer-die-niedrigepraevalenz-der-ambulanten-peritonealdialyse-in-deutschland.35

Gemeinsamer Bundesausschuss G-BA - Innovationsausschuss. MEHIRA - Gestuftes Versorgungsmodell zur Förderung der mentalen Gesundheit von Flüchtlingen (Mental Health in Refugees and Asylum Seekers): Projektbeschreibung 2021; Stand: 21.01.2021]. Im Internet: https:// innovationsfonds.g-ba.de/projekte/versorgungsforschung/mehira-gestuftes-versorgungsmodell-zur-foerderung-der-mentalen-gesundheit-vonfluechtlingen-mental-health-in-refugees-and-asylum-seekers.60

Gemeinsamer Bundesausschuss G-BA - Innovationsausschuss. MULTIqual - Entwicklung und Validierung von Qualitätsindikatoren für Multimorbidität: Projektbeschreibung 2021; Stand: 21.01.2021] Im Internet: https://innovationsfonds.g-ba.de/projekte/versorgungsforschung/multiqual-entwicklung-und-validierung-von-qualitaetsindikatoren-fuer-multimorbiditaet.57

Gemeinsamer Bundesausschuss G-BA - Innovationsausschuss. MVP-STAT - Bedarfsgerechtigkeit der medizinischen Versorgung Pflegebedürftiger in stationären Einrichtungen: Projektbeschreibung 2021; Stand: 21.01.2021]. Im Internet: https:// innovationsfonds.g-ba.de/projekte/versorgungsforschung/ mvp-stat-bedarfsgerechtigkeit-der-medizinischen-versorgung-pflegebeduerftiger-in-stationaeren-einrichtungen.38

Gemeinsamer Bundesausschuss G-BA - Innovationsausschuss. NierenTx $360^{\circ}$ - Bessere Versorgung, besseres Überleben, bessere Wirtschaftlichkeit nach Nierentransplantation: Projektbeschreibung 2021; Stand: 21.01.2021]. Im Internet: https:// innovationsfonds.g-ba.de/projekte/neue-versorgungsformen/ nierentx3600-bessere-versorgung-besseres-ueberleben-bessere-wirtschaftlichkeit-nach-nierentransplantation.64 
Gemeinsamer Bundesausschuss G-BA - Innovationsausschuss. NoMiG - Notfallversorgung von Migranten und Geflüchteten: Projektbeschreibung 2021; Stand: 21.01.2021]. Im Internet: https:// innovationsfonds.g-ba.de/projekte/versorgungsforschung/ nomig-notfallversorgung-von-migranten-und-gefluechteten. 37 Gemeinsamer Bundesausschuss G-BA - Innovationsausschuss. Notfallund Akutversorgung Brandenburg - Bestandsaufnahme und Weiterentwicklung der Notfall- und Akutversorgung im Land Brandenburg: Projektbeschreibung 2021; Stand: 21.01.2021]. Im Internet: https:// innovationsfonds.g-ba.de/projekte/versorgungsforschung/notfall-undakutversorgung-brandenburg-bestandsaufnahme-und-weiterentwicklung-der-notfall-und-akutversorgung-im-land-brandenburg.61

Gemeinsamer Bundesausschuss G-BA - Innovationsausschuss. NPPV - Verbesserte Versorgung psychischer und neurologischer Erkrankungen: Projektbeschreibung 2021; Stand: 21.01.2021]. Im Internet: https://innovationsfonds.g-ba.de/projekte/neue-versorgungsformen/ nppv-verbesserte-versorgung-psychischer-und-neurologischer-erkrankungen.69

Gemeinsamer Bundesausschuss G-BA - Innovationsausschuss. NWGA - NetzWerk GesundAktiv: Projektbeschreibung 2021; Stand: 21.01.2021]. Im Internet: https://innovationsfonds.g-ba.de/projekte/ neue-versorgungsformen/nwga-netzwerk-gesundaktiv.91

Gemeinsamer Bundesausschuss G-BA - Innovationsausschuss. OSA-PSY - Optimierung der stationären Arzneimitteltherapie bei psychischen Erkrankungen: Projektbeschreibung 2021; Stand: 21.01.2021]. Im Internet: https://innovationsfonds.g-ba.de/projekte/ versorgungsforschung/osa-psy-optimierung-der-stationaeren-arzneimitteltherapie-bei-psychischen-erkrankungen. 9

Gemeinsamer Bundesausschuss G-BA - Innovationsausschuss. P-AK - Psychotherapeutische Abendklinik: Neue Versorgungsform für depressive Patienten: Projektbeschreibung 2021; Stand: 21.01.2021] Im Internet: https://innovationsfonds.g-ba.de/projekte/ versorgungsforschung/p-ak-psychotherapeutische-abendklinik-neueversorgungsform-fuer-depressive-patienten.59

Gemeinsamer Bundesausschuss G-BA - Innovationsausschuss. PAV - Patientensicherheit in der Ambulanten Versorgung: Projektbeschreibung 2021; Stand: 21.01.2021]. Im Internet: https:// innovationsfonds.g-ba.de/projekte/versorgungsforschung/ pav-patientensicherheit-in-der-ambulanten-versorgung. 15

Gemeinsamer Bundesausschuss G-BA - Innovationsausschuss. PAWEL - Patientensicherheit, Wirtschaftlichkeit und Lebensqualität: Reduktion von Delirrisiko und postoperativer kognitiver Dysfunktion (POCD) nach Elektivoperationen im Alter: Projektbeschreibung 2021; Stand: 21.01.2021]. Im Internet: https:// innovationsfonds.g-ba.de/projekte/versorgungsforschung/ pawel-patientensicherheit-wirtschaftlichkeit-und-lebensqualitaetreduktion-von-delirrisiko-und-postoperativer-kognitiver-dysfunktionpocd-nach-elektivoperationen-im-alter.16

Gemeinsamer Bundesausschuss G-BA - Innovationsausschuss. PeriAge - Optimierung der perioperativen Versorgung älterer Patienten: Projektbeschreibung 2021; Stand: 21.01.2021]. Im Internet: https://innovationsfonds.g-ba.de/projekte/versorgungsforschung/periage-optimierung-der-perioperativen-versorgung-aelterer-patienten.56

Gemeinsamer Bundesausschuss G-BA - Innovationsausschuss. PIM-STOP - Vergleich der prädiktiven Validität von Instrumenten zur Bestimmung potenziell inadäquater Medikation bei Älteren: Projektbeschreibung 2021; Stand: 21.01.2021]. Im Internet: https:// innovationsfonds.g-ba.de/projekte/versorgungsforschung/ pim-stop-vergleich-der-praediktiven-validitaet-von-instrumentenzur-bestimmung-potenziell-inadaequater-medikation-bei-aelteren. 12

Gemeinsamer Bundesausschuss G-BA - Innovationsausschuss. PINA - Folgeschäden nach prolongierter Intensivbehandlung: Entwicklung und Pilotierung einer Intensiv-Nachsorgeambulanz: Projektbeschrei- bung 2021; Stand: 21.01.2021]. Im Internet" https:// innovationsfonds.g-ba.de/projekte/versorgungsforschung/ pina-folgeschaeden-nach-prolongierter-intensivbehandlung-entwicklung-und-pilotierung-einer-intensiv-nachsorgeambulanz.55

Gemeinsamer Bundesausschuss G-BA - Innovationsausschuss. PRÄZIS - Prävention des Zervixkarzinoms und dessen Vorstufen bei Frauen im Saarland: Projektbeschreibung 2021; Stand: 21.01.2021]. Im Internet: https://innovationsfonds.g-ba.de/projekte/versorgungsforschung/praezis-praevention-des-zervixkarzinoms-und-dessenvorstufen-bei-frauen-im-saarland. 49

Gemeinsamer Bundesausschuss G-BA - Innovationsausschuss. PrimA-QuO - Optimierte primärärztliche Versorgung von Kindern und Jugendlichen mit psychischen Auffälligkeiten und Störungen: Projektbeschreibung 2021; Stand: 21.01.2021]. Im Internet: https:// innovationsfonds.g-ba.de/projekte/versorgungsforschung/prima-quooptimierte-primaeraerztliche-versorgung-von-kindern-und-jugendlichen-mit-psychischen-auffaelligkeiten-und-stoerungen.31

Gemeinsamer Bundesausschuss G-BA - Innovationsausschuss. ProFem - Versorgung, Funktionsfähigkeit und Lebensqualität nach proximaler Femurfraktur: Projektbeschreibung 2021; Stand: 21.01.2021]. Im Internet: https://innovationsfonds.g-ba.de/projekte/ versorgungsforschung/profem-versorgung-funktionsfaehigkeit-undlebensqualitaet-nach-proximaler-femurfraktur.42

Gemeinsamer Bundesausschuss G-BA - Innovationsausschuss. ProMeKa - Ausmaß und Trends der problematischen Medikation von Benzodiazepinen, Z-Substanzen, Opioid-Analgetika und Antidepressiva bei Kassenpatienten: Projektbeschreibung 2021; Stand: 21.01.2021]. Im Internet: https://innovationsfonds.g-ba.de/projekte/ versorgungsforschung/promeka-ausmass-und-trends-der-problematischen-medikation-von-benzodiazepinen-z-substanzen-opioidanalgetika-und-antidepressiva-bei-kassenpatienten. 48

Gemeinsamer Bundesausschuss G-BA - Innovationsausschuss. PROMISE - Prozessoptimierung durch interdisziplinäre, sektorenübergreifende Versorgung am Beispiel von Hüft- und Kniearthrosen: Projektbeschreibung 2021; Stand: 21.01.2021]. Im Internet: https:// innovationsfonds.g-ba.de/projekte/neue-versorgungsformen/ promise-prozessoptimierung-durch-interdisziplinaere-sektorenuebergreifende-versorgung-am-beispiel-von-hueft-und-kniearthrosen. 83

Gemeinsamer Bundesausschuss G-BA - Innovationsausschuss. PRO-ONK_ROUTINE - Entwicklung eines Kurzinstruments zur Messung gesundheitsbezogener Lebensqualität bei Krebspatienten und Analyse der Implementierung: Projektbeschreibung 2021; Stand: 21.01.2021]. Im Internet: https://innovationsfonds.g-ba.de/ projekte/versorgungsforschung/pro-onk-routine-entwicklung-eineskurzinstruments-zur-messung-gesundheitsbezogener-lebensqualitaet-bei-krebspatienten-und-analyse-der-implementierung.24

Gemeinsamer Bundesausschuss G-BA - Innovationsausschuss. PROPERmed - Entwicklung eines Instruments zur Identifikation von multimorbiden Hochrisikopatienten für negative Folgen von Multimedikation: Projektbeschreibung 2021; Stand: 21.01.2021]. Im Internet: https://innovationsfonds.g-ba.de/projekte/versorgungsforschung/propermed-entwicklung-eines-instruments-zur-identifikation-von-multimorbiden-hochrisikopatienten-fuer-negative-folgenvon-multimedikation. 18

Gemeinsamer Bundesausschuss G-BA - Innovationsausschuss. PsychCare - Wirksamkeit sektorenübergreifender Versorgungsmodelle in der Psychiatrie - eine prospektive, kontrollierte multizentrische Beobachtungsstudie: Projektbeschreibung 2021; Stand: 21.01.2021]. Im Internet" https://innovationsfonds.g-ba.de/projekte/ versorgungsforschung/psychcare-wirksamkeit-sektorenuebergreifender-versorgungsmodelle-in-der-psychiatrie-eine-prospektive-kontrollierte-multizentrische-beobachtungsstudie. 52

Gemeinsamer Bundesausschuss G-BA - Innovationsausschuss. PV-Monitor - Nutzung von Routinedaten zur Pharmakovigilanz in Deutschland: Methodenentwicklung und erste Anwendungen: 
Projektbeschreibung 2021; Stand: 21.01.2021]. Im Internet: https:// innovationsfonds.g-ba.de/projekte/versorgungsforschung/ pv-monitor-nutzung-von-routinedaten-zur-pharmakovigilanz-indeutschland-methodenentwicklung-und-erste-anwendungen.20

Gemeinsamer Bundesausschuss G-BA - Innovationsausschuss. QS-Notfall - Verbesserung der Notfallversorgung von Herzinfarktpatienten in Berlin und Brandenburg: Projektbeschreibung 2021; Stand: 21.01.2021], Im Internet: https://innovationsfonds.g-ba.de/ projekte/versorgungsforschung/qs-notfall-verbesserung-der-notfallversorgung-von-herzinfarktpatienten-in-berlin-und-brandenburg. 11

Gemeinsamer Bundesausschuss G-BA - Innovationsausschuss. RECOVER - Modell der sektorenübergreifend-koordinierten, schweregradgestuften, evidenzbasierten Versorgung psychischer Erkrankungen: Projektbeschreibung 2021; Stand: 21.01.2021]. Im Internet: https://innovationsfonds.g-ba.de/projekte/neue-versorgungsformen/recover-modell-der-sektorenuebergreifend-koordinierten-schweregradgestuften-evidenzbasierten-versorgung-psychischer-erkrankungen. 85

Gemeinsamer Bundesausschuss G-BA - Innovationsausschuss. RESIST - Resistenzvermeidung durch adäquaten Antibiotikaeinsatz bei akuten Atemwegserkrankungen: Projektbeschreibung 2021; Stand: 21.01.2021]. Im Internet: https://innovationsfonds.g-ba.de/projekte/ neue-versorgungsformen/resist-resistenzvermeidung-durch-adaequaten-antibiotikaeinsatz-bei-akuten-atemwegserkrankungen.77

Gemeinsamer Bundesausschuss G-BA - Innovationsausschuss. ReToCdiff - Reduktion von Infektionen mit Toxin-bildenden Clostridium difficile in Geriatrischen Kliniken: Projektbeschreibung 2021; Stand: 21.01.2021]. Im Internet: https:// innovationsfonds.g-ba.de/projekte/versorgungsforschung/ retocdiff-reduktion-von-infektionen-mit-toxin-bildenden-clostridium-difficile-in-geriatrischen-kliniken. 58

Gemeinsamer Bundesausschuss G-BA - Innovationsausschuss. Rheuma-VOR - Verbesserung der rheumatologischen Versorgungsqualität durch koordinierte Kooperation: Projektbeschreibung 2021; Stand: 21.01.2021]. Im Internet: https://innovationsfonds.g-ba.de/ projekte/neue-versorgungsformen/rheuma-vor-verbesserung-derrheumatologischen-versorgungsqualitaet-durch-koordinierte-kooperation. 89

Gemeinsamer Bundesausschuss G-BA - Innovationsausschuss. Rise-uP - Rücken innovative Schmerztherapie mit e-Health für unsere Patienten: Projektbeschreibung 2021; Stand: 21.01.2021]. Im Internet: https://innovationsfonds.g-ba.de/projekte/neue-versorgungsformen/rise-up-ruecken-innovative-schmerztherapie-mit-ehealth-fuer-unsere-patienten.72

Gemeinsamer Bundesausschuss G-BA - Innovationsausschuss. SAVOIR - Evaluierung der SAPV-Richtlinie: Outcomes, Interaktionen, Regionale Unterschiede: Projektbeschreibung 2021; Stand: 21.01.2021]. Im Internet: https://innovationsfonds.g-ba.de/projekte/ versorgungsforschung/savoir-evaluierung-der-sapv-richtlinie-outcomes-interaktionen-regionale-unterschiede. 5

Gemeinsamer Bundesausschuss G-BA - Innovationsausschuss. SEAL - Strukturierte Früh-Erkennung einer Asymptomatischen Leberzirrhose in Rheinland-Pfalz und im Saarland: Projektbeschreibung 2021; Stand: 21.01.2021]. Im Internet: https:// innovationsfonds.g-ba.de/projekte/neue-versorgungsformen/ seal-strukturierte-frueh-erkennung-einer-asymptomatischen-leberzirrhose-in-rheinland-pfalz-und-im-saarland.74

Gemeinsamer Bundesausschuss G-BA - Innovationsausschuss. TeleDerm - Implementierung teledermatologischer Konsile in die hausärztliche Versorgung - kontrollierte Studie mit qualitativ-quantitativer Prozessevaluation: Projektbeschreibung 2021; Stand: 21.01.2021]. Im Internet: https://innovationsfonds.g-ba.de/projekte/ neue-versorgungsformen/telederm-implementierung-teledermatologischer-konsile-in-die-hausaerztliche-versorgung-kontrolliertestudie-mit-qualitativ-quantitativer-prozessevaluation.67
Gemeinsamer Bundesausschuss G-BA - Innovationsausschuss. TeleDermatologie - Allgemein-, Fach- und Notfallmedizin im ländlichen Raum am Beispiel Dermatologie: Projektbeschreibung 2021; Stand: 21.01.2021]. Im Internet: https:// innovationsfonds.g-ba.de/projekte/neue-versorgungsformen/ teledermatologie-allgemein-fach-und-notfallmedizin-im-laendlichenraum-am-beispiel-dermatologie. 65

Gemeinsamer Bundesausschuss G-BA - Innovationsausschuss. Telenotarzt Bayern - Pilotprojekt zur telemedizinischen Unterstützung der Notfallversorgung im Rettungsdienst einer ländlich strukturierten Region: Projektbeschreibung 2021; Stand: 21.01.2021]. Im Internet: https://innovationsfonds.g-ba.de/projekte/ neue-versorgungsformen/telenotarzt-bayern-pilotprojekt-zur-telemedizinischen-unterstuetzung-der-notfallversorgung-im-rettungsdienst-einer-laendlich-strukturierten-region.68

Gemeinsamer Bundesausschuss G-BA - Innovationsausschuss. TELE-QOL - Settingsensitive Konzeptualisierung und Erfassung der Lebensqualität in der telemedizinischen Versorgung: Projektbeschreibung 2021; Stand: 21.01.2021]. Im Internet: https:// innovationsfonds.g-ba.de/projekte/versorgungsforschung/ tele-qol-settingsensitive-konzeptualisierung-und-erfassung-derlebensqualitaet-in-der-telemedizinischen-versorgung. 27

Gemeinsamer Bundesausschuss G-BA - Innovationsausschuss. Telnet@NRW - Telemedizinisches, intersektorales Netzwerk als neue digitale Struktur zur messbaren Verbesserung der wohnortnahen Gesundheitsversorgung: Projektbeschreibung 2021; Stand: 21.01.2021]. Im Internet: https://innovationsfonds.g-ba.de/projekte/ neue-versorgungsformen/telnetatnrw-telemedizinisches-intersektorales-netzwerk-als-neue-digitale-struktur-zur-messbaren-verbesserung-der-wohnortnahen-gesundheitsversorgung. 84

Gemeinsamer Bundesausschuss G-BA - Innovationsausschuss. TransFIT - Frühintervention zur Vorbereitung und Begleitung des Transitionspro- zesses aus der Kinder- und Jugendmedizin in die Erwachsenenmedizin: Projektbeschreibung 2021; Stand: 21.01.2021]. Im Internet: https://innovationsfonds.g-ba.de/projekte/ neue-versorgungsformen/transfit-fruehintervention-zur-vorbereitung-und-begleitung-des-transitionsprozesses-aus-der-kinder-undjugendmedizin-in-die-erwachsenenmedizin. 87

Gemeinsamer Bundesausschuss G-BA - Innovationsausschuss. TRANSLATE-NAMSE - Verbesserung der Versorgung von Menschen mit seltenen Erkrankungen durch Umsetzung von im nationalen Aktionsplan (NAMSE) konsentierten Maßnahmen: Projektbeschreibung 2021; Stand: 21.01.2021]. Im Internet: https:// innovationsfonds.g-ba.de/projekte/neue-versorgungsformen/ translate-namse-verbesserung-der-versorgung-von-menschen-mitseltenen-erkrankungen-durch-umsetzung-von-im-nationalen-aktionsplan-namse-konsentierten-massnahmen.78

Gemeinsamer Bundesausschuss G-BA - Innovationsausschuss. VaMB - Value Stream Mapping in Brustzentren - Ein Lösungsansatz zur Optimierung des Entlassungsprozesses: Projektbeschreibung 2021; Stand: 21.01.2021]. Im Internet: https://innovationsfonds.g-ba.de/ projekte/versorgungsforschung/vamb-value-stream-mapping-inbrustzentren-ein-loesungsansatz-zur-optimierung-des-entlassungsprozesses. 39

Gemeinsamer Bundesausschuss G-BA - Innovationsausschuss. VERhO - Versorgung von Menschen mit Rheuma optimieren: Projektbeschreibung 2021; Stand: 21.01.2021]. Im Internet: https:// innovationsfonds.g-ba.de/projekte/neue-versorgungsformen/ verho-versorgung-von-menschen-mit-rheuma-optimieren.80

Gemeinsamer Bundesausschuss G-BA - Innovationsausschuss. ViDiKi - Virtuelle Diabetesambulanz für Kinder und Jugendliche: Projektbeschreibung 2021; Stand: 21.01.2021]. Im Internet: https:// innovationsfonds.g-ba.de/projekte/neue-versorgungsformen/ vidiki-virtuelle-diabetesambulanz-fuer-kinder-und-jugendliche.90 
Gemeinsamer Bundesausschuss G-BA - Innovationsausschuss. WASH - Evaluation eines web-assistierten Selbsthilfe-Trainings für Eltern von Kindern mit Aufmerksamkeitsdefizit- / Hyperaktivitätsstörung: Projektbeschreibung 2021; Stand: 21.01.2021]. Im Internet :https:// innovationsfonds.g-ba.de/projekte/versorgungsforschung/wash-evaluation-eines-web-assistierten-selbsthilfe-trainings-fuer-eltern-vonkindern-mit-aufmerksamkeitsdefizit-hyperaktivitaetsstoerung.30

Geraedts M, Mehl C, Schmitz J et al. Entwicklung eines Indikatorensets zur Evaluation der Integrierten Versorgung Gesundes Kinzigtal. Zeitschrift für Evidenz, Fortbildung und Qualität im. Gesundheitswesen 2020; 150-152: 54-64

Gerber C.. Evaluation des Versorgungsprojekts „Neurologisch-psychiatrische und psychotherapeutische Versorgung (NPPV)" - ein neues Versorgungskonzept in der Region Nordrhein 2019

Gerber C, Pollmanns J. Folgeevaluation der Versorger_innen im Versorgungsprojekt „Neurologisch-psychiatrische und psychotherapeutische Versorgung (NPPV)“. 2019; 2020.

Golchert J, Roehr S, Berg F et al. HELP@APP: development and evaluation of a self-help app for traumatized Syrian refugees in Germany - a study protocol of a randomized controlled trial. BMC Psychiatry 2019; 19 (1): 131

Graf K. Ergebnisbericht. 2020; Im Internet: https://innovationsfonds. g-ba.de/downloads/projekt-dokumente/36/2020-12-18_TelenotarztBayern_Ergebnisbericht.pdf Stand: 08.02.2021]

Grasemann C, Matar N, Bauer J et al. Ein strukturierter Versorgungspfad von der Pädiatrie in die Erwachsenenmedizin für Jugendliche und junge Erwachsene mit einer seltenen Erkrankung. Monatsschrift Kinderheilkunde 2020

Großgarten K. Verbesserte Versorgung psychischer und neurologischer Erkrankungen. 2020;Im Internet https://www.drks.de/drks_ web/navigate.do? navigationld = trial.HTML\&TRIAL ID = DRKS00022754 Stand: 15.20.2021].

Großgarten K, Bergmann F, Grimmeisen S.. Neuropsychiatrische und psychotherapeutische Versorgung (NPPV). 2017

Härter M. Evaluation des Vertrages zur Versorgung in den Fachgebieten der Neurologie, Psychiatrie, Psychosomatik und Psychotherapie in Baden-Württemberg gem. §73c SGB V. 2017; Im Internet https:// www.drks.de/drks_web/navigate.do?navigationld = trial. HTML\&TRIAL_ID = DRKS00013114 Stand: 15.02.2021] .

Hasebrook J, Huebner J, Metelmann C et al. Projekt LandRettung: Schnelle Hilfe im Notfall. f \& w Führen und Wirtschaften im Krankenhaus 2019; 1: 36-9

Hefner $G$, Hahn M, Toto $S$ et al. Potentially inappropriate medication in older psychiatric patients. European Journal of Clinical Pharmacology 2021; 77 (3): 331-9

Hefner G, Wolff J, Hahn M et al. Prevalence and sort of pharmacokinetic drug-drug interactions in hospitalized psychiatric patients. Journal of Neural Transmission 2020; 127 (8): 1185-98.

Hermann A, Aust E.. Reader response: An observational study on quality of life and preferences to sustain life in locked-in state. Neurology 2020; 95 (6): 275

Hildebrandt H.. INTEGRAL - 10 Jahres Evaluation der Integrierten Versorgung Gesundes Kinzigtal in Aufbau- und Konsolidierungsphase. 2018; Im Internet: https://www.drks.de/drks_web/navigate. do? navigationld = trial.. HTML\&TRIAL_ID = DRKS00012804; Stand: 15.02.2021].

Hommel T. Wie ViDiKi Kindern mit Diabetes hilft; o. J. Im Internet: https://www.springermedizin.de/gesundheitspolitik/telemedizinwie-vidiki-kindern-mit-diabetes-hilft $/ 15417134$ ? searchResult $=2$. Virtuelle \%20Diabetesambulanz\%20f\%C3\%BCr\%20Kinder\%20 und $\% 20$ Jugendliche $\% 20$ \%20ViDiKi\&searchBackButton = true; Stand: 08.02.2021]
Jacob I, Bruch L, Heinrich F et al. Erstversorgung von Herzinfarktpatienten - Daten eines vom Innovationsfonds geförderten Projektes zu Diagnosesicherheit und konsekutiver Versorgungszeit. 2019

Joos S, Koch R. Implementierung teledermatologischer Konsile in die hausärztliche Versorgung - eine kontrollierte Studie mit qualitativquantitativer Prozessevaluation. 2017; Im Internet: https://www. drks.de/drks_web/navigate.do?navigationld = trial.HTML\&TRIAL_ ID = DRKS00012944; Stand: 15.02.2021].

Kamradt M, Kaufmann-Kolle P, Andres E et al. Sustainable reduction of antibiotic-induced antimicrobial resistance (ARena) in German ambulatory care: study protocol of a cluster randomised trial. Implementation science: IS 2018; 13 (1): 23

Kathmann W.. Rückenschmerz: Projekt „RiSe-uP“ des Schmerznetzes Bayern am Start. Schmerzmedizin 2017; 33 (6): 21

Kaulke K, Ihle P, Drepper J et al. Datenschutzkonzept für das Projekt Qualitätssicherung - Notfallversorgung von Herzinfarktpatienten in Berlin und in den Brandenburgischen Landkreisen Havelland und Oberhavel (QS-Notfall). 2019;

Kerkemeyer L, Goncalves A, SchiessI C et al. Implementierung eines integrierten Rückenschmerzenversorgungskonzeptes - eine Beobachtungsstudie mit Primär- und Sekundärdaten (Rise-uP, Rücken innovative Schmerztherapie mit e-Health für unsere Patienten). 2018;

Kirsch C, Doyle I-M, Krause O et al. Lessons learned“ - Herausforderungen im Rekrutierungsprozess in der cluster-randomisierten Pflegeheimstudie „HIOPP-3 iTBX“. Zeitschrift für Evidenz, Fortbildung und Qualität im. Gesundheitswesen 2020; 156-157: 24-32

Kirsch C, Junius-Walker U, Doyle I-M et al. „Vier gewinnt“ - die Herausforderung eines mehrstufigen Rekrutierungsverfahrens in der Cluster-randomisierten Studie „HIOPP-3 iTBX“. 2019

Koch R, Haumann H, Sturm $\mathrm{H}$ et al. Implementierung teledermatologischer Konsile in die hausärztliche Versorgung - eine kontrollierte Studie mit qualitativ-quantitativer Prozessevaluation (TeleDerm) 2017

Koch R, Polanc A, Haumann $\mathrm{H}$ et al. Improving cooperation between general practitioners and dermatologists via telemedicine: study protocol of the cluster-randomized controlled TeleDerm study. Trials 2018; 19 (1): 583

Koch R, Polanc A, Joos S. Chancen und Herausforderungen der Digitalisierung am Beispiel des TeleDerm-Projekts - Ergebnispräsentation und Selbsterfahrung. 2019

Körner M, Dinius J, Manser T et al. Entwicklung und Erprobung eines komplexen interprofessionellen Trainingsprogramms zur Verbesserung der Patientensicherheit (KOMPAS). 2017;

Krause O. HIOPP-3-iTBX: Angemessene und sichere Medikation für Heimbewohner/innen mithilfe einer interprofessionellen Toolbox (AMTS-Toolbox). 2018; Im Internet: https://www.drks.de/drks_web/ navigate.do? navigationld $=$ trial. HTML\&TRIAL_ID = DRKS00013588 Stand: 15.02.2021].

Krause O, Wiese B, Doyle I-M et al. Multidisciplinary intervention to improve medication safety in nursing home residents: protocol of a cluster randomised controlled trial (HIOPP-3-iTBX study). BMC Geriatrics 2019; 19 (1): 24

Kucher R. Erweiterte koordinierte ärztliche Pflegeheimversorgung. 2017; Im Internet: https://www.drks.de/drks_web/navigate. do? navigationld = trial.HTML\&TRIAL_ID = DRKS00012703 ;Stand 15.02.2021].

Laag S, Kellermann-Mühlhoff P, Beckmann T et al. Das Projekt Anwendung für ein digital unterstütztes Arzneimitteltherapie-Management - AdAM. G\&S Gesundheits- und Sozialpolitik 2017; 71 (1): 22-6

Lambert M, Karow A, Gallinat J et al. Study protocol for a randomised controlled trial evaluating an evidence-based, stepped and coordinated care service model for mental disorders (RECOVER). BMJ Open 2020; 10 (5): e036021 
Löffler C, Iwen J, Krüger A et al. RESIST - ein neues Versorgungskonzept zur Resistenzvermeidung. HNO Nachrichten 2019; 49 (5): 24-8

Löffler C, Krüger A, Daubmann A et al. Optimizing Antibiotic Prescribing for Acute Respiratory Tract Infection in German Primary Care: Study Protocol for Evaluation of the RESIST Program. JMIR Res Protoc 2020; 9 (9): e18648-e18648

Ludwig-Maximilians-Universität München. Accountable Care in Deutschland. Verbesserung der Patientenversorgung durch mehr Vernetzung und informierten Dialog; o. J. Stand: 08.02.2021]. Im Internet: https://acd-projekt.de/wp-content/uploads/2018/06/ Poster_ACD_neu.pdf

Magaard J, Liebherz S, Melchior $\mathrm{H}$ et al. Evaluation des Vertrages zur Versorgung in den Fachgebieten der Neurologie, Psychiatrie, Psychosomatik und Psychotherapie in Baden-Württemberg gemäß $\S 73 c$ SGB V. 2018

Magaard J, Seeralan T, Ramona M et al. Evaluation eines Vertrages zur Versorgung in den Fachgebieten der Neurologie, Psychiatrie, Psychosomatik und Psychotherapie in Baden-Württemberg aus der Sicht der teilnehmenden Behandlerinnen und Behandler. 2019

Maier B, Kühne A, Minden $\mathrm{H}$ et al. Verbesserung der Notfallversorgung von Herzinfarktpatienten in Berlin und Brandenburg (QS-Notfall). 2017

Malonga Makosi D, Toni I, König J et al. KiDSafe - Evaluation einer Intervention zur Verbesserung der ambulanten Versorgung von Kindern und Jugendlichen mit Arzneimitteln durch Erhöhung der Arzneimitteltherapiesicherheit. 2020

Marschall U, Spies C.. Das Projekt Enhanced Recovery after Intensive Care - ERIC. G\&S Gesundheits- und Sozialpolitik 2017; 71 (1): 32-4

Marx G, Greiner W.. Telemedizinisches, intersektorales Netzwerk als neue digitale Gesund-heitsstruktur zur messbaren Verbesserung der wohnortnahen Versorgung (TELnet@NRW). Evaluationsdesign. Im Internet: https://www.telnet.nrw/wp-content/uploads/TELnet@ NRW_Evaluationsdesign-Analyseplan.pdf; Stand: 08.02.2021]

Marx G.. Telemedical, Intersectoral Network as New Digital Health Structure to Measurably Improve the Local Health Care (TELnet@ NRW). 2017; Im Internet" https://clinicaltrials.gov/ct2/show/study/ NCT03137589; Stand: 15.02.2021].

Mehl C, Koester I, Graf E et al. Operationalisierung von Qualitätsindikatoren aus Routinedaten am Beispiel der Evaluation der Integrierten Versorgung Gesundes Kinzigtal. 2020

Mehl C, Schmitz J, Ihle P et al. Entwicklung eines Indikatoren-Sets für die Evaluation der Integrierten Versorgung Gesundes Kinzigtal. 2018;

Meier U. NPPV - Versorgungsmodell in Nordrhein. NeuroTransmitter 2018; 29 (S1): 20-1

Metelmann C, Metelmann B, Kohnen D et al. Evaluation of a Rural Emergency Medical Service Project in Germany: Protocol for a Multimethod and Multiperspective Longitudinal Analysis. JMIR Res Protoc 2020; 9 (2): e14358-e14358

Moormann T. BGM-innovativ: Betriebliches Gesundheits- und Versorgungs- management nachhaltig gestalten. G\&S Gesundheitsund Sozialpolitik 2017; 71 (1): 17-21

Müller B, González-González A, Klaaßen-Mielke R et al. Wirksamkeit der ,Anwendung für ein digital unterstütztes ArzneimitteltherapieManagement (AdAM) ' - Studienprotokoll der Cluster-randomisierten kontrollierten Studie. 2019;

Müller C, Fleischmann N, Tetzlaff B et al. ,interprof ACT“: Effekte von Strategien zur Verbesserung ärztlich-pflegerischer Zusammenarbeit auf Krankenhausaufnahmen von Pflegeheimbewohnerinnen und -bewohnern - Studiendesign einer clusterrandomisierten, kontrollierten Studie. 2017;

Müller C, Fleischmann N, Tetzlaff B et al. "interprofACT"-Effekte von Strategien zur Verbesserung ärztlich-pflegerischer Zusammenarbeit auf Krankenhausaufnahmen von Pflegeheimbewohnerinnen und -bewohnern: Studiendesign der clusterrandomisierten, kontrollierten Studie. 2017

Müller C, Hesjedal-Streller B, Fleischmann N et al. Effects of strategies to improve general practitioner-nurse collaboration and communication in regard to hospital admissions of nursing home residents (interprof ACT): study protocol for a cluster randomised controlled trial. Trials 2020; 21 (1): 913

Muth C. Application for an Electronic Medication Management Support System (AdAM). 2018; Im Internet: https://clinicaltrials.gov/ ct2/show/NCT03430336 Stand: 08.20.2021].

Nagel M, Arslanow A, Nguyen-Tat M et al. SEAL program - Early detection of liver fibrosis and cirrhosis by screening of the general population.

Nagl M, Luck T, Renner A et al. HELP@APP - Entwicklung und Evaluation einer Selbsthilfe-App für traumatisierte syrische Flüchtlinge in Deutschland. 2017

Nelles G.. Versorgungsprojekt NPPV erfolgreich etabliert. NeuroTransmitter 2019; 30 (6): 14-5

Neubert A.. KiDSafe - Verbesserung der Arzneimitteltherapiesicherheit bei Kindern und Jugendlichen: Implementierung und Evaluation einer neuen Versorgungsform. 2018; Im Internet: https://www.drks. de/drks_web/navigate.do?navigationld = trial.HTML\&TRIAL_ ID = DRKS00013924; Stand: 15.02.2021].

Neubert A, Schwab M, Rascher W et al. Verbesserung der Versorgung von Kindern-und Jugendlichen mit Arzneimitteln durch Erhöhung der Arzneimittelsicherheit - Das KiDSafe Projekt. 2017

Neubert A, Schwab M, Riedel C et al. Improving the care of children and adolescents with medicines by enhancing medication safety - The KiDSafe Project. 2017;

Neumann A, Soltmann B, Peter S von et al. PsychCare: Wirksamkeit sektorenübergreifender Versorgungsmodelle in der Psychiatrie - eine prospektive, kontrollierte multizentrische Beobachtungsstudie 2017

Nguyen-Tat M, Lammert F, Arslanow A et al. SEAL-Programm - Früherkennung der Leberzirrhose durch Screening der Allgemeinbevölkerung im Rahmen des Check-up 35: 2018

Nöhre M, Bauer-Hohmann M, Klewitz F et al. Prevalence and Correlates of Cognitive Impairment in Kidney Transplant Patients Using the DemTect-Results of a KTx360 Substudy. Frontiers in Psychiatry 2019; 10: 791

Nöhre M, Schieffer E, Hanke A et al. Obesity After Kidney Transplantation-Results of a KTx360 ${ }^{\circ}$ Substudy. Frontiers in Psychiatry 2020; 11: 399

o. V. Verordnungsrate von Antibiotika durch RESIST gesunken. 2020; Im Internet: https://www.aerzteblatt.de/nachrichten/116484; Stand: 08.02.2021].

Olotu C, Lebherz L, Härter M et al. Improvement of perioperative care of the elderly patient (PeriAge): protocol of a controlled interventional feasibility study. BMJ Open 2019; 9 (11): e031837

Ortwein A, Mehl C, Siegel A et al. Modellierung regionaler Indikatoren für die Evaluation Integrierter Versorgung am Beispiel Gesundes Kinzigtal. 2019

Pape L, Zwaan M, de, Tegtbur U et al. The KTx $360^{\circ}$-study: a multicenter, multisectoral, multimodal, telemedicine-based follow-up care model to improve care and reduce health-care costs after kidney transplantation in children and adults. BMC Health Services Research 2017; 17 (1): 587

Pape L. Kidney Transplantation $360^{\circ}$. 2017; Im Internet https://www. isrctn.com/ISRCTN29416382' Stand: 15.02.2021].

Paul N, Grunow J, Weiß B et al. Enhanced Recovery after Intensive Care - ERIC“. Der Anaesthesist 2020; 69 (12): 937-9

Paul N, Dähnert E, Grunow J et al. Blended-Learning-Konzepte in der Intensivmedizin am Beispiel des ERIC-Trainings. Anästhesiol Intensivmed Notfallmed Schmerzther 2021; 56 (1): 29-40 
Peth J, Schulz H, König H-H. Evaluation of a Cross-sectional Coordinated, Severity Stepped, Evidence-based Care Model for Mental Disorders (RECOVER). 2018; Im Internet: https://www. clinicaltrials.gov/ct2/show/ NCT03459664?term $=$ RECOVER\&cond $=$ mental + illness\&cntry $=$ DE\&draw $=2 \&$ rank $=1$; Stand: 15.02 .2021$]$. Pfaff H.. BGM-innovativ - Arbeitsplatznahes, trägerübergreifendes Versorgungsmanagement der Betriebskrankenkassen. 2018; Im Internet: https://www.drks.de/drks_web/navigate.do?navigationld = trial. HTML\&TRIAL_ID = DRKS00015120; Stand: 15.02.2021].

Pfennig A.. Wirksamkeit sektorenübergreifender Versorgungsmodelle in der Psychiatrie - eine prospektive, kontrollierte multizentrische Beobachtungsstudie. 2020; Im Internet: https://www.drks.de/ drks_web/navigate.do?navigationld = trial.HTML\&TRIAL_ ID = DRKS000

22535; Stand: 15.02.2021]

Polanc A, Koch R, Thies C et al. Implementierung teledermatologischer Konsile in Hausarztpraxen: Erste Ergebnisse aus dem Innovationsfondsprojekt TELEDerm. 2019;

Poss-Doering R, Kühn L, Kamradt M. Fostering Appropriate Antibiotic Use in a Complex Intervention: Mixed-Methods Process Evaluation Alongside the Cluster-Randomized Trial ARena. Antibiotics (Basel) 2020; 9 (12): 878

Poss-Doering R, Kamradt M, Glassen K et al. Promoting rational antibiotic prescribing for non-complicated infections: understanding social influence in primary care networks in Germany. BMC Fam Pract 2020; 21 (1): 51

Poss-Doering R, Kamradt M, Stuermlinger A et al. The complex phenomenon of dysrational antibiotics prescribing decisions in German primary healthcare: a qualitative interview study using dual process theory. Antimicrob Resist Infect Control 2020; 9 (1): 6

Prasser C, Süss R, Hahnenkamp K et al. Der Telenotarzt als Innovation des Rettungsdienstes im ländlichen Raum - Kosten der Implementierung. Gesundheitsökonomie \& Qualitätsmanagement 2020; 25 (3): 150-6

Priebe J, Haas K, Moreno Sanchez L et al. Digital Treatment of Back Pain versus Standard of Care: The Cluster-Randomized Controlled Trial, Rise-uP. J Pain Res 2020; 13 1823-38.

PROGNOS AG.. Gesamtevaluation des Innovationsfonds: Teilbericht über die erste Evaluationsphase. 2019;Stand: 19.03.2021]. Im Internet: https://www.prognos.com/de/projekt/gesamtevaluationdes-innovationsfonds

Radevic-Pahl N, Bauer I, Laub O et al. Optimierte primärärztliche Versorgung von Kindern und Jugendlichen mit psychischen Auffälligkeiten und Störungen - Evaluation von Qualität und Outcomes (PrimA-QuO). 2017

Rebecca Beerheide. RESIST: Dialog über Antibiotika-Verordnung. Deutsches Ärzteblatt 2018 Stand: 08.02.2021]; 115(15):691 red. VERhO-Projekt verlängert. Orthop. Rheuma 2019; 22 (3): 10 Riedel-Heller S.. Entwicklung und Evaluation einer Selbsthilfe-App für traumatisierte syrische Flüchtlinge. 2018; Im Internet: https://www. drks.de/drks_web/navigate.do?navigationld = trial.HTML\&TRIAL_ ID = DRKS00013782 Stand: 15.02.2021].

Rohr M, Brunnthaler V, Brandstetter S et al. PINA-Studie: Konzept für eine Intensiv-Nachsorgeambulanz zur Verbesserung der gesundheitsbezogenen Lebensqualität nach einem verlängerten Intensivaufenthalt. 2020

Röhr S, Jung F, Renner A et al. Recruitment and Baseline Characteristics of Participants in the "Sanadak" Trial: A Self-Help App for Syrian Refugees with Post-traumatic Stress. Int J Environ Res Public Health 2020; $17: 20$

Rüffer F, Neumann A, Peter S von et al. Entwicklung von Qualitätsindikatoren für eine psychiatrische Versorgung. 2019
Sánchez A, Thomas C, Deeken F et al. Patient safety, cost-effectiveness, and quality of life: reduction of delirium risk and postoperative cognitive dysfunction after elective procedures in older adults-study protocol for a stepped-wedge cluster randomized trial (PAWEL Study). Trials 2019; 20 (1): 71

Scheithauer S, Kaase M, Eiffert $\mathrm{H}$ et al. ReToCdiff - Reduktion von Infektionen mit Toxin-bildenden Clostridium difficile in Geriatrischen Kliniken. 2017;

Schmidt C.. Eine randomisierte kontrollierte Studie zur Untersuchung eines integrierten Versorgungskonzepts (NICC) bei chronischen Herzerkrankungen: CardioCare MV. 2017; Im Internet: https://www. drks.de/drks_web/navigate.do?navigationld = trial.HTML\&TRIAL_ ID = DRKS00013124; Stand: 15.02.2021].

Schmidt C.. Investigation of a Novel Integrated Care Concept (NICC) for Patients Suffering From Chronic Cardiovascular Disease (CardioCare MV). 2017; Im Internet: https://clinicaltrials.gov/ct2/ show/NCT03317951; Stand: 15.02.2021].

Schmidt C, Hillebrandt B, Mann M.. HerzEffekt MV - Ein innovatives Versorgungsmodell für Herzpatienten 2017

Schmidt C, Öner A, Mann M et al. A novel integrated care concept (NICC) versus standard care in the treatment of chronic cardiovascular diseases: protocol for the randomized controlled trial CardioCare MV. Trials 2018; 19 (1): 120

Schmitt J, Eberlein-Gonska M, Kuhlen R.. Ergebnisbericht. 2020; Im Internet: https://innovationsfonds.g-ba.de/downloads/projekt-dokumente/20/2020-10-09_IMPRESS_Ergebnisbericht.pdf; Stand: 08.02.2021]

Schmitt ], Rößler M, Scriba P et al. Auswirkungen des IQM-PeerReview-Verfahrens auf die Mortalität von Patienten mit Beatmung > $24 \mathrm{~h}$ : Ergebnisse einer cluster-randomisierten, kontrollierten Studie (IMPRESS). 2020

Schmitt J, Schoffer O, Walther $F$ et al. Effectiveness of the IQM peer review procedure to improve in-patient care-a pragmatic cluster randomized controlled trial (IMPRESS): study design and baseline results. Journal of Public Health 2019

Schoffer O, Roessler M, Walther F et al. Patient-Level and HospitalLevel Risk Factors for In-Hospital Mortality in Patients Ventilated for More Than 24 Hours: Results of a Nationwide Cohort Study. Journal of Intensive Care Medicine 2020; 0885066620942182

Schoffer O, Rößler M, Walther $\mathrm{F}$ et al. Patienten- und krankenhausspezifische Einflussfaktoren auf die Mortalität von Patienten mit Beatmung > 24 Stunden: eine explorative Multilevel-Analyse im Rahmen der IMPRESS-Studie. 2019

Schubert I, Geraedts M, Gröne O et al. 10-Jahres-Evaluation der populationsbezogenen Integrierten Versorgung Gesundes Kinzigtal - Aufbau- und Konsolidierungsphase (INTEGRAL). 2017

Schubert I, Graf E, Siegel A et al. 10-Jahres-Evaluation der populationsbezogenen Integrierten Versorgung Gesundes Kinzigtal - INTEG RAL. 2020

Schubert I, Siegel A, Graf E et al. Study protocol for a quasi-experimental claims-based study evaluating 10 -year results of the population-based integrated healthcare model 'Gesundes Kinzigtal' (Healthy Kinzigtal): the INTEGRAL study. BMJ Open 2019; 9 (1): e025945

Schubert I, Graf E, Siegel A et al. Ergebnisbericht. Im Internet" https://innovationsfonds.g-ba.de/downloads/projekt-dokumente/38/2020-12-18_INTEGRAL_Ergebnisbericht.pdf; Stand: 08.02.2021]

Schulz M, Krüger A, Wollny A et al. Erste Ergebnisse der Evaluation der neuen Versorgungsform RESIST - zur Resistenzvermeidung durch adäquaten Antibiotikaeinsatz bei akuten Atemwegsinfektionen 2019

Schwarting A, Dreher M, Assmann G et al. Erfahrungen und Ergebnisse aus Rheuma-VOR. Zeitschrift für Rheumatologie 2019; 78 (8): 743-52. 
Schwarting A. Von ADAPTHERA zu Rheuma-VOR: Konzept der koordinierten Kooperation zur Verbesserung der rheumatologischen Versorgungsqualität. Aktuelle Rheumatologie 2018; 43 (5): 406-9

Schwarting A, Dreher M, Assmann G et al. Rheuma-VOR: Verbesserung der rheumatologischen Versorgungsqualität durch koordinierte. Kooperation - Ein Update. 2020;

Sengbusch von S, Doerdelmann J, Lemke $S$ et al. Parental expectations before and after 12-month experience with video consultations combined with regular outpatient care for children with type 1 diabetes: a qualitative study. Diabet. Med. 2020; 00 (): e14410

Sengbusch 5 von.. Virtuelle Diabetesambulanz für Kinder und Jugendliche - ViDiKi. 2017; Im Internet: https://www.drks.de/drks_ web/navigate.do? navigationld = trial.HTML\&TRIAL_

ID = DRKS00012645; Stand: 15.02.2021].

Sengbusch von S, Eisemann N, Mueller-Godeffroy E et al. Outcomes of monthly video consultations as an add-on to regular care for children with type 1 diabetes: A 6-month quasi-randomized clinical trial followed by an extension phase. Pediatr Diabetes 2020; 21 (8): 1502-15.

Sengbusch S, von, Forster A.. Das Projekt Virtuelle Diabetesambulanz für Kinder und Jugendliche - ViDiKi. GuS 2017; 71 (1): 27-31

Söling S, Köberlein-Neu J, Müller B et al. From sensitization to adoption? A qualitative study of the implementation of a digitally supported intervention for clinical decision making in polypharmacy. Implementation science: IS 2020; 15 (1): 82

Soltmann B, Neumann A, Peter S von et al. Wirksamkeit sektorenübergreifender Versorgungsmodelle in der Psychiatrie (PsychCare): erste Ergebnisse der Primärdatenerhebung. 2019

Spies C. Enhanced Recovery After Intensive Care (ERIC). 2018; Im Internet: https://clinicaltrials.gov/ct2/show/NCT03671447; Stand: 15.20.2021].

Splieth C. Ergebnisbericht. Im Internet: https:// innovationsfonds.g-ba.de/downloads/projekt-dokumente/34/2020-12-18_IpKiSuN_Ergebnisbericht.pdf; Stand: 03.02.2021]

Stolz R, Doyle I-M, Junius-Walker U et al. Forschungsprojekt HIOPP-3: Hausärztliche Initiative zur Optimierung der Patientensicherheit bei Polypharmazie - Ergebnisse der Analyse struktureller Qualitätsmerkmale der beteiligten Akteure. 2020

Stolz R, Haumann H, Joos S et al. HIOPP-3: Interprofessional optimization of medication in nursing home residents - a cluster randomized controlled trial. 2018

Strotbaum V.. Wie nehmen Ärzte und Patienten Telemedizin wahr? Ergebnisse einer Akzeptanzbefragung im Rahmen des Innovationsfonds-Projektes „TELnet@NRW“. 2020;

Strotbaum V. TELnet@NRW - Implementierung eines telemedizinischen, intersektoralen Netzwerks als digitale Gesundheitsstruktur zur Verbesserung der wohnortnahen Versorgung. 2017
Stuermlinger A, Poss-Doering R, Glassen K. Dealing with patient expectations regarding the prescription of antibiotics in ambulatory care in Germany: A qualitative analysis. Research Square. 2021;

Sturm H, Wildermuth R, Stolz R et al. Diverging Awareness of Postoperative Delirium and Cognitive Dysfunction in German Health Care Providers. Clin Interv Aging 2019; 14: 2125-35.

Sturm H, Wildermuth R, Stolz R et al. Differences in awareness between physicians and nurses for postoperative delirium and persistent cognitive dysfunction in geriatric patients. Int J Integr Care 2019; 19 (4): 455

Sundmacher L. Accountable Care in Deutschland. 2020; Im Internet https://www.drks.de/drks_web/navigate.do?navigationld = trial. HTML\&TRIAL_ID = DRKS00020884; Stand: 15.02.2021].

Szecsenyi J. Sustainable reduction of antibiotics-induced antimicrobial resistance (ARena) in German ambulatory care. 2017; Im Internet https://www.isrctn.com/ISRCTN58150046 Stand: 15.02.2021].

Tölle T.. Rücken innovative Schmerztherapie mit e-Health für unsere Patienten. 2018; Im Internet: https://www.drks.de/drks_web/ navigate. do? navigationld = trial.HTML\&TRIAL_ID = DRKS00015048 Stand: 15.02.2021].

Toni I, Moritz K, Schulze C et al. KiDSafe - Implementierung einer neuen Versorgungsform zur Verbesserung der ambulanten Versorgung von Kindern und Jugendlichen mit Arzneimitteln durch Erhöhung der Arzneimitteltherapiesicherheit. 2020;

Walther F.. Effectiveness of a collegial consultation procedure to improve in-patient care - a pragmatic cluster randomized controlled trial. 2018;Im Internet https://www.isrctn.com/ISRCTN10188560 Stand: 15.02.2021].

Warth von der R, Kaiser V, Reese C.. Implementation of a complex health services intervention in long-term care homes: a process evaluation using focus groups. Research Square. 2020;

Weber J, Angermaier A, Bollweg K.. Acute neurological care in north-east Germany with telemedicine support (ANNOTeM): protocol of a multi-center, controlled, open-label, two-arm intervention study. BMC Health Services Research 2020; 20 (1): 755

Weiss B, Paul N, Kraufmann B et al. Qualitätssteigerung in der Intensivmedizin durch Telemedizin: Beispiel ERIC. Anästhesiol Intensivmed Notfallmed Schmerzther 2021; 56 (1): 41-51

Ziegler A, Mann M, Brandewiede B et al. Statistical analysis plan for the randomized controlled trial CardioCare MV investigating a novel integrated care concept (NICC) for patients suffering from chronic cardiovascular disease. Trials 2020; 21 (1): 131 